\title{
Genome-wide transcriptome profiling of transgenic hop (Humulus lupulus L.) constitutively overexpressing HMRKY1 and HMDR1 transcription factors
}

Ajay Kumar Mishra ${ }^{1}$, Ganesh Selvaraj Duraisamy ${ }^{1}$, Mudra Khare ${ }^{1}$, Tomáš Kocábek', Jernej Jakse², Jindřich Bříza ${ }^{1}$, Josef Patzak ${ }^{3}$, Teruo Sano ${ }^{4}$ and Jaroslav Matoušek ${ }^{1 *}$

\begin{abstract}
Background: The hop plant (Humulus lupulus L.) is a valuable source of several secondary metabolites, such as flavonoids, bitter acids, and essential oils. These compounds are widely implicated in the beer brewing industry and are having potential biomedical applications. Several independent breeding programs around the world have been initiated to develop new cultivars with enriched lupulin and secondary metabolite contents but met with limited success due to several constraints. In the present work, a pioneering attempt has been made to overexpress master regulator binary transcription factor complex formed by HNRKY1 and HNDR1 using a plant expression vector to enhance the level of prenylflavonoid and bitter acid content in the hop. Subsequently, we performed transcriptional profiling using high-throughput RNA-Seq technology in leaves of resultant transformants and wild-type hop to gain in-depth information about the genome-wide functional changes induced by HMRKY1 and HNDR1 overexpression.
\end{abstract}

Results: The transgenic WW-lines exhibited an elevated expression of structural and regulatory genes involved in prenylflavonoid and bitter acid biosynthesis pathways. In addition, the comparative transcriptome analysis revealed a total of 522 transcripts involved in 30 pathways, including lipids and amino acids biosynthesis, primary carbon metabolism, phytohormone signaling and stress responses were differentially expressed in WW-transformants. It was apparent from the whole transcriptome sequencing that modulation of primary carbon metabolism and other pathways by HNRRKY1 and HNDR1 overexpression resulted in enhanced substrate flux towards secondary metabolites pathway. The detailed analyses suggested that none of the pathways or genes, which have a detrimental effect on physiology, growth and development processes, were induced on a genome-wide scale in WW-transgenic lines.

Conclusions: Taken together, our results suggest that HNRKY1 and HNDR1 simultaneous overexpression positively regulates the prenylflavonoid and bitter acid biosynthesis pathways in the hop and thus these transgenes are presented as prospective candidates for achieving enhanced secondary metabolite content in the hop.

Keywords: Bitter acids, Flavonoids, Genetic transformation, Humulus lupulus, Secondary metabolite, Transcription factors, Transcriptome analysis

\footnotetext{
* Correspondence: jmat@umbr.cas.cz

${ }^{1}$ Biology Centre of the Czech Academy of Sciences, Institute of Plant

Molecular Biology, Department of Molecular Genetics, Branišovská 31, 37005

České Budějovice, Czech Republic

Full list of author information is available at the end of the article
}

(c) The Author(s). 2018 Open Access This article is distributed under the terms of the Creative Commons Attribution 4.0 International License (http://creativecommons.org/licenses/by/4.0/), which permits unrestricted use, distribution, and reproduction in any medium, provided you give appropriate credit to the original author(s) and the source, provide a link to the Creative Commons license, and indicate if changes were made. The Creative Commons Public Domain Dedication waiver (http://creativecommons.org/publicdomain/zero/1.0/) applies to the data made available in this article, unless otherwise stated. 


\section{Background}

Hop (Humulus lupulus L.) is a herbaceous, perennial climbing vine, a dioecious plant belonging to the Cannabaceae family, widely cultivated throughout the temperate regions of the world for the brewing industry as a source of flavour-active secondary metabolites, bitter acids, and shelf-life stabilizer. In addition, hop extracts and/or metabolome has received considerable attention in pharmaceutical applications due to their diverse biological properties, such as anti-carcinogenic [1], anti-inflammatory [2], estrogenic [3], sedative [4], antimicrobial [5] and antioxidation [6] activities. The female plants of hop produce cone-like inflorescences, commonly referred to as "hop cones" or "hops" contain a large number of highly metabolically active glandular trichomes (lupulin glands) on the inner side of bracts and bracteoles, which synthesize and/or secret specific secondary metabolites such as essential oils, bitter acids (humulone or $\alpha$-acid and lupulone or $\beta$-acid) and prenylated flavonoids (xanthohumol and desmethylxanthohumol) during its phased maturation [7, 8]. The bracts represent the aggregation of modified leaves, which makes up the outer structure of female cones. In addition to cones, lupulin glands are also sparsely distributed on the undersides of leaves (Additional file 1: Figure S1), contain detectable levels of hop acids [9], terpenes [10], xanthohumol [11] and flavonols [12] and thus serve as the primary site of secondary metabolite accumulation [11]. Hop plants undergo different phenological growth stages and reaches peak maturity at around its third year of growth. Generally, after a three-year of normal growth, the hop cones completely develop and ripe with highest metabolome content [13].

The shikimate pathway serves as the primary source of biosynthesis of flavonoid and other phenylpropanoid precursors in plants. At the link between primary and secondary metabolism, phenylalanine ammonia-lyase (PAL) or tyrosine ammonia lyase (TAL) catalyze the non-oxidative deamination of phenylalanine to trans-cinnamate and direct the carbon flow from the shikimate pathway to the various branches of the general phenylpropanoids and flavonoids biosynthesis [14]. The other two enzymes cinnamate 4-hydroxylase $(\mathrm{C} 4 \mathrm{H})$, and 4-coumaroyl CoA-Ligase (4CL) catalyze other two committed successive steps leading to 4-coumaroyl-CoA substrate, which represents the prime branch point for all subsequent phenylpropanoid branches and flavonoid biosynthesis in plants [14]. The terminal step of prenylated flavonoids in hop cones is mediated by chalcone synthase CHS_H1 [15], prenyltransferase (PRT) [16] and O-methyltransferase 1 (OMT1) [11] enzymes with the involvement of several types of transcription factors, belonging to MYB, bHLH, WDR and WRKY families in either independent or combinatorial manner (Fig. 1) [17-20]. The parallel activation of CHS_H1 promoter has been shown to be driven by either highly organized ternary
MBW complexes (Hls-Myb3/HlbHLH2/HlWDR1 or $H l \mathrm{MYB} 2 / H l \mathrm{bHLH} 2 / H l \mathrm{WDR} 1)$ or binary complexes (HlbHLH2 /HlWDR1) through protein-protein interactions (Fig. 1) $[18,19]$. Recently we have cloned and characterized HIWRKY1 (homolog of AtWRKY75) transcription factor which forms a binary complex with WD40 repeat protein1 (HlWDR1) and acts as a master transcription factor to activate structural genes of terminal steps and ternary MBW complex of the prenylflavonoid (PF) and bitter acids (BA) biosynthesis pathways [18]. The expression of HlWRKY1 transcription factor can be activated by a protein kinase, modulated by autoactivation and dependent on RNA silencing machinery [19].

The HIWRKY1 and HlWDR1 transcription factors are preferentially expressed in lupulin glands, function as a master regulator to drive the PF and BA biosynthesis pathways $[18,19]$. The homologs of these two transcription factors have multifunctional roles [21, 22]. In this context, it was imperative to understand whether constitutive expression of these two transcription factors could serve as an important strategy to enhance the PF and BA content by analyzing overall impact over the morphological, developmental attributes and other related pathways of hop. The several independent and cooperative hop breeding programmes around the world have been directed towards the development of new and improved cultivars with advantageous traits such as higher yield, enriched lupulin, high metabolome content to satisfy the demand of the brewing industry [13, 23]. However, conventional breeding methods have been largely constrained by the sexual incompatibility of developed lines, limited genetic resources, long cumbersome process and appearance of unintended characteristics [24]. Nevertheless, alternative direction involving the transformation of hop, utilizing either heterologous or homologous gene expression system may provide a promising approach for secondary metabolite engineering [25]. The genetic transformation technology, which is considered as an extension of conventional plant breeding technologies has been practiced in several plant species for the introduction of desirable agronomic traits from more than three decades and became an established technology to generate precise, rapid and stable modifications in utilized cultivars [26, 27]. Over the past years, genetic engineering technology has been used for manipulation of secondary metabolite biosynthesis in different plant species via constitutive overexpression of homologous and heterologous transcription factors [28-30].

The integration of transgene cassettes into the host genome could alter the expression of adjacent and downstream genes from the insertion site and owing to different feedback regulatory mechanism disruption of single gene activity alter the expression of hundreds or thousands of other genes [31]. Generally, genetically modified plants have been 


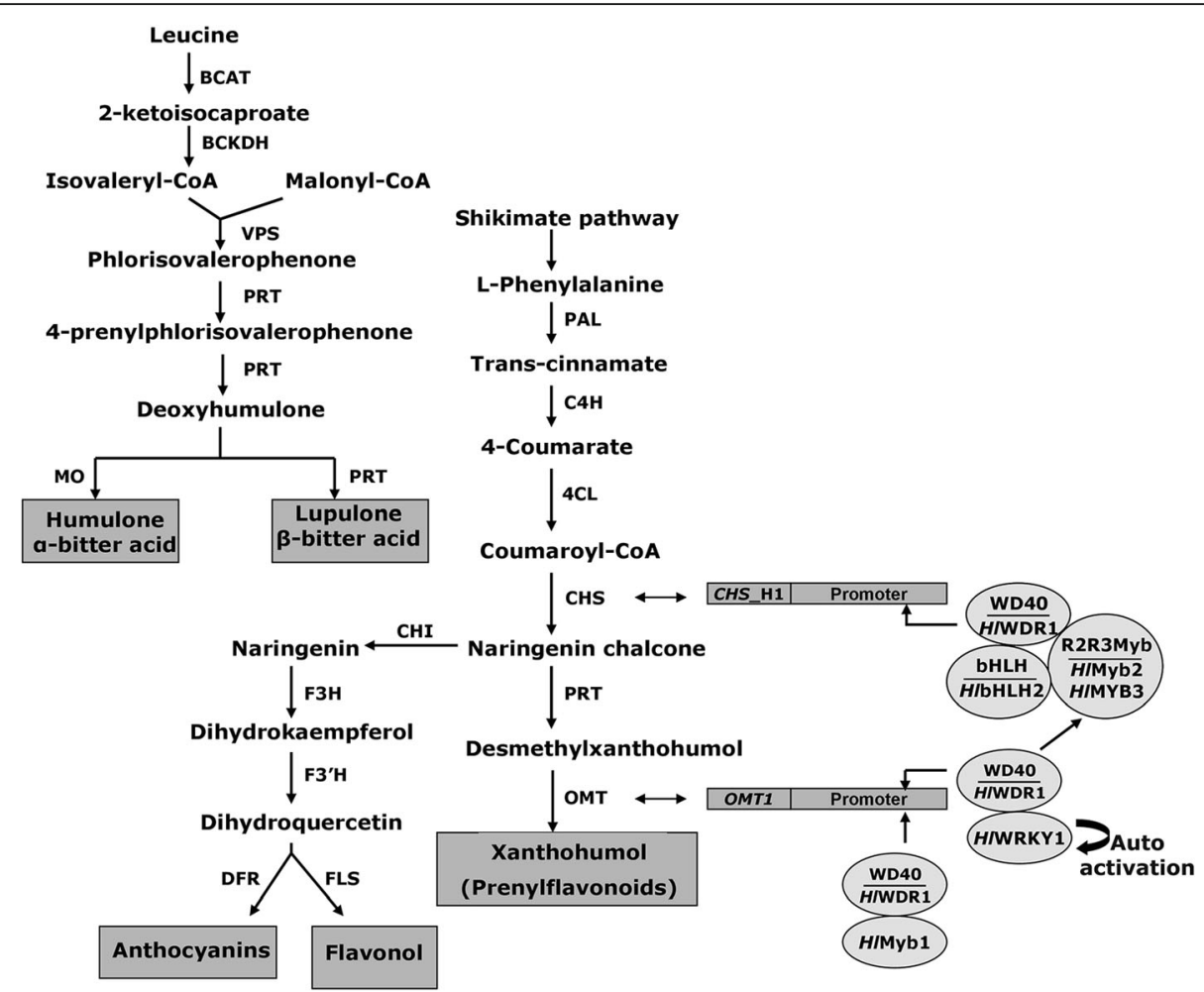

Fig. 1 An overview of the bitter acid, phenylpropanoids and flavonoid biosynthesis pathways in the hop. The main intermediate compounds are shown with the abbreviation of respective enzymatic steps. Enzyme abbreviations are PAL: phenylalanine ammonia lyase; $\mathrm{C} 4 \mathrm{H}$ : cinnamate 4hydroxylase, 4CL: coumarate coenzyme A ligase, CHS: chalcone synthase, PRT: prenyltransferase; OMT: O-methyltransferases; VPS: valerophenone synthase, BCAT: branched chain aminotransferase; BCKDH: branched-chain alpha-ketoacid dehydrogenase; MO: monooxygenases; $\mathrm{CHI}$ : chalcone flavanone isomerase; F3H: flavanone 3-hydroxylase; F3'H, flavonoid 3'-hydroxylase; DFR, dihydroflavonol 4-reductase; FLS: flavonol synthase. OMTI and $C H S \_H 1$ represent gene isoforms of O-methyltransferases and chalcone synthase genes, respectively in the hop

evaluated based on comparative metabolomics, proteomics and nutritional composition analyses [32]. Remarkably, a genomics and transcriptomics technique has been recommended by the European Food Safety Agency (EFSA) as an additional evaluation criterion to improve the breadth of comparative analysis [32].

In this regard, recent revolutionary advances in nextgeneration sequencing technologies, in conjunction with the refined computational tools provide rapid, cost-effective generation of transcriptomics resources and more accurate analysis of differentially expressed genes between transgenic plants and their wild-type equivalents [33]. Over the past decade, several studies were conducted based on high-throughput sequencing of plant transcriptome, which provided better and broader understanding of the molecular level information on various model and non model plant species such as olive [34], cucumber [35], chickpea [36], tomato [37], potato [38], sweet potato [39] and many more. These studies provided valuable molecular and genetic information, including the discovery of molecular marker and novel genes, insight into genetic network, transcriptional and posttranscriptional gene regulation and metabolic pathways to accelerate crop improvement program through breeding and genetic engineering techniques $[40,41]$. To this end, the comparative transcriptome analysis serves as an integrated approach to provide insights into the molecular basis underlying specific biological events, profiling of changes in gene expression levels under specific experimental or environmental conditions and detect unique alternatively spliced isoforms of transcripts [42]. Moreover, the comparative transcriptome analyses have been widely used to examine the unintended pleiotropic effects in a number of transgenic plant systems by comparing them with their isogenic counterpart [43-46].

In this study, transgenic hop lines overexpressing binary transcription factors complex HIWRKY1 and HlWDR1 have been developed. The developed hop transgenic lines have been used further to understand the impact of transgenic events on transcriptome expression profiles in a leaf of red-bine Czech Osvald'72 cultivar of hop, which is well known for its unique metabolome content and composition. The comparative transcriptome analysis suggests that the overexpression of HIWRKY1 and HIWDR1 leads to upregulation of 
transcription factors and structural genes involved in PF and BA biosynthesis pathways, including differential regulations of genes involved in diverse biological processes in the hop. To our knowledge, our study represents one of the first pioneering efforts to enhance the expression level of genes of PF and BA biosynthetic pathway in the hop with significant evidence at the transcriptional level that genetically modified hop is not harmful concerning the biosafety of genetically modified organisms (GMOs).

\section{Methods}

\section{Hop transformation and screening of transgenic lines}

The full-length gene sequence of HlWRKY1 (GenBank accession no: FR751557) and HIWDR1 (GenBank accession no: FN689721) were amplified from our previously constructed lupulin-specific cDNA library using gene-specific primers (Additional file 2: Table S1) and cloned into dual expression cassette vector WWpPCV91 using adapter ligation-mediated PCR strategy as described previously [19]. The generated construct harboring HlWRKY1 cDNA was fused to the tetramer of enhancer of cauliflower mosaic virus (CaMV) $35 \mathrm{~S}$ promoter and HIWDR1 cDNA was fused to mannopine synthase bidirectional promoter, respectively (Fig. 2) and was transferred into the A. tumefaciens strain GV3101 by electroporation [47]. The single colony of A. tumefaciens with the plasmid WWpPCV91 was inoculated in liquid Luria-Bertani medium (LB; $0.5 \% \mathrm{NaCl}$, $1 \%$ yeast extract and $1.6 \%$ tryptone) supplemented with $100 \mathrm{mgl}^{-1}$ carbenicillin and $50 \mathrm{mgl}^{-1}$ kanamycin. The bacterial cultures were grown until the optical density reached at $600 \mathrm{~nm}\left(\mathrm{OD}_{600}\right)$ of 0.6 at $28^{\circ} \mathrm{C}$ with $200 \mathrm{rpm}$. The culture was diluted 1:50 into fresh LB medium with $200 \mu \mathrm{M}$ acetosyringone (AS) and incubated at $28^{\circ} \mathrm{C}$ until an $\mathrm{OD}_{600}$ of 0.6. The bacterial suspension was centrifuged at $6000 \mathrm{~g}$ for $10 \mathrm{~min}$ at $4{ }^{\circ} \mathrm{C}$ and bacterial pellet was suspended in MTA medium (10 mM $\mathrm{MgSO}_{4}, 0.1 \%$ Tween 20, $200 \mu \mathrm{M}$ AS) with an $\mathrm{OD}_{600}$ of 1.0 for transformation. The in vitro-derived internode stem segment explants (5-100 $\mathrm{mm}$ in length) of hop (cv. Osvald's 72) were submerged into the bacterial suspension for $20 \mathrm{~min}$. The infected explants were washed with sterile water and after wiping excessive water, the explants were cultured onto regeneration $\mathrm{R}$ medium (Murashige and Skoog basal medium containing $20 \mathrm{~g} \mathrm{l}^{-1}$ glucose, $1.0 \mathrm{mg} \mathrm{l}^{-1}$ zeatin, $0.25 \mathrm{mg} \mathrm{l}^{-1}$ IAA, $6 \mathrm{~g} \mathrm{l}^{-1}$ plant agar) supplemented with $200 \mu \mathrm{M}$ AS for 3 days at $22-24{ }^{\circ} \mathrm{C}$. Afterward, explants were transferred onto $\mathrm{R}$ medium containing $250 \mathrm{mg} \mathrm{l}^{-1}$ Timentin and $1.5 \mathrm{mg} \mathrm{l}^{-1}$ hygromycin $\mathrm{B}$ and were cultured under $16 \mathrm{~h}$ photoperiod at $22-24{ }^{\circ} \mathrm{C}$ for $4-6$ weeks, until the ostentation of shoots. Regenerated shoots (Additional file 3: Figure S2A) were transferred to a rooting $\mathrm{S}$ medium (Murashige and Skoog basal medium supplemented with $20 \mathrm{~g} \mathrm{l}^{-1}$ glucose, $250 \mathrm{mg} \mathrm{l}^{-1}$ Timentin, $1.5 \mathrm{mg} \mathrm{l}^{-1}$ hygromycin B,
$6 \mathrm{~g} \mathrm{l}^{-1}$ plant agar) impregnated with exogenous hormone supplements of IAA $\left(1.0 \mathrm{mg} \mathrm{l}^{-1}\right)$ and IBA (1.0 $\mathrm{mg} \mathrm{l}^{-1}$ ) following previously described protocol [48]. To screen putative transgenic lines (WW) co-expressing $H l W R K Y 1$ and $H l W D R 1$ transcription factors, Southern blot and PCR analyses were performed. Genomic DNA was extracted from leaves of WW-transformant and wild-type (WT) hop plant using a previously described protocol [49]. For the genomic DNA hybridization analysis, $15 \mu \mathrm{g}$ of genomic DNA was digested overnight either with BglII or PacI according to supplier's instruction (New England Biolabs, MA, USA), separated on a $1 \%$ agarose gel at $25 \mathrm{~V}$ overnight with TBE buffer and transferred onto a positively charged nylon membrane (Qiabrane Nylon Plus) according to the manufacturer's specifications (Qiagen, Hilden, Germany). The DNA probes (HPT and HlWDR1) were radiolabelled with $\alpha-{ }_{-}^{32}$ P-labelled dCTP using Rediprime ${ }^{\mathrm{mm}}$ random prime labeling kit (Amersham Pharmacia Biotech, Freiburg, Germany) according to the manufacturer's protocol. Pre-hybridization and hybridization reactions were carried out at $65^{\circ} \mathrm{C}$ following a previously described protocol [50]. Membranes were analyzed by autoradiography (Typhoon 9200 PhosphoImager, USA). The PCR analysis was performed using WWpPCV91 vector specific forward and gene-specific reverse primer (Additional file 2: Table S1).

The independent WW-transformant and WT-lines were transferred from in vitro to in vivo growth chamber conditions at $22{ }^{\circ} \mathrm{C}$ with a $16 \mathrm{~h}$ photoperiod for gradual acclimatization and to ensure their survival (Additional file 3: Figure S2B). The successfully acclimatized three-month-old well rooted three WW-transformants (B11, B23, and B24) and wild-type (WT) plantlets were transferred to larger pots containing soil-vermiculite mixture (3:1) and grown under greenhouse conditions (Additional file 3: Figure S2C). The visual observations were taken regularly to evaluate growth and morphological characteristics (height, number of nodes, lupulin glands density, and leaf morphology). In addition, chlorophyll content was evaluated according to Lichtenthaler and Wellburn method [51]. The absorbance of the extract recorded at 646 and $663 \mathrm{~nm}$ wavelengths by using UV- spectrophotometry (Shimadzu, Japan).

\section{RNA extraction, high-throughput RNA sequencing and assembly generation}

The leaves were harvested in the second year growing season of WW-transformants and WT-hop plants (28 months after the shifting to the greenhouse) for total RNA preparation. Total RNA was extracted from $100 \mathrm{mg}$ of young leaves of control and three screened WW-transgenic lines (B11, B23, and B24) using Concert ${ }^{\mathrm{Tm}}$ Plant RNA Purification Reagent (Invitrogen), followed by RNA purification and DNA contamination removal using DNA-free ${ }^{\text {Tx }}$ DNA Removal kit (Ambion, USA) according to the manufacturer's 


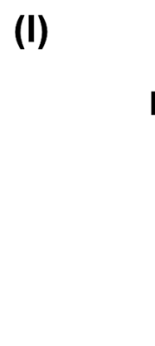

(II)

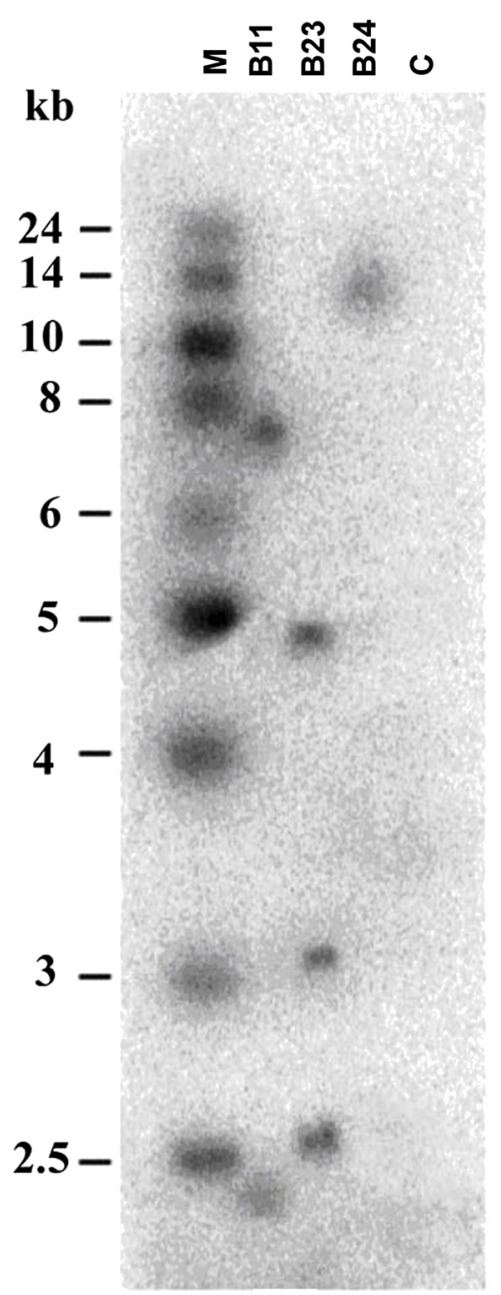

(A)

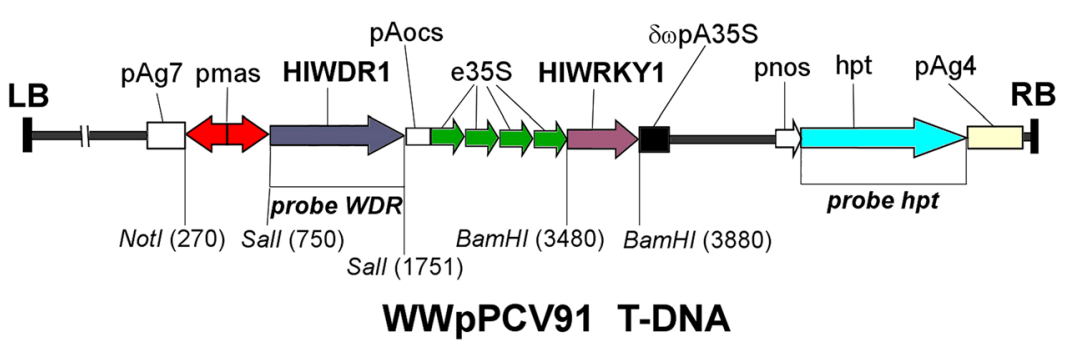

(8858 bp)

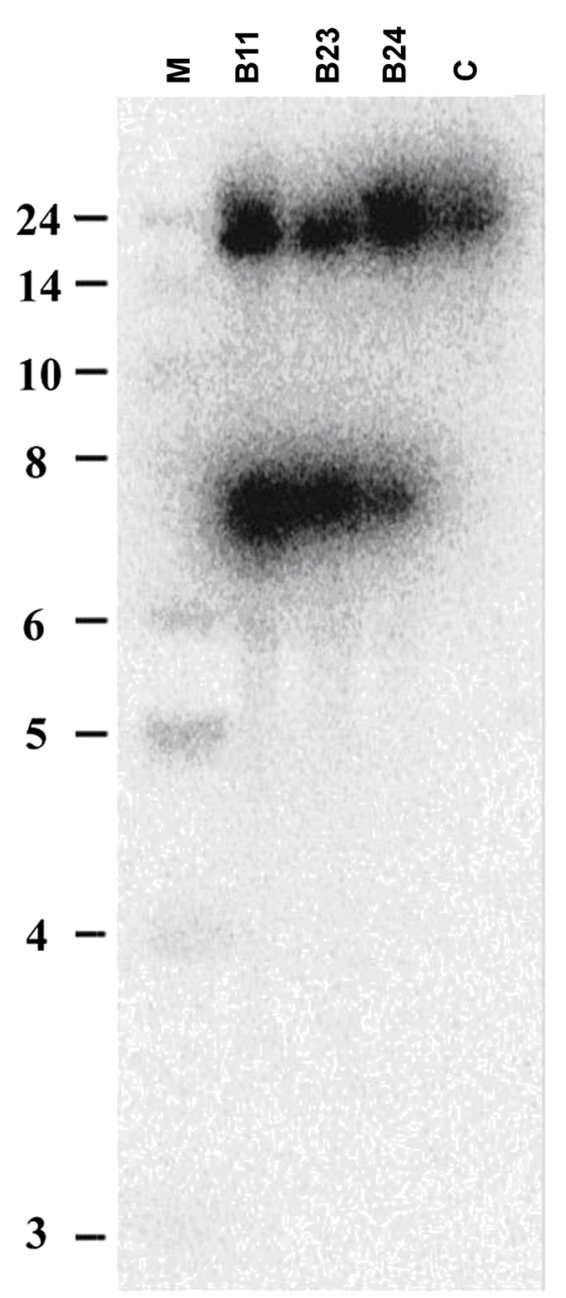

(B)

Fig. 2 Molecular analysis of hop transgenic lines overexpressing HMRKY1 and HNRD1 transcription factors. (I) Schematic representation of the T-DNA region of the plant expression vector WWpPCV91, used for hop transformation.LB: left border, pmas: mannopine synthase bidirectional promoter, HPT: hygromycin phosphotransferase gene, e35S - enhancer of the $35 \mathrm{~S}$ promoter from CaMV virus, pnos: nopaline synthase promoter, RB: right border. (II) Southern blot of hop genomic DNA isolated from WW-transgenic lines (B1 1, B23, B24) and control plants. The hybridization was performed with HPT (a) and WDR probe (b). The DNA markers (1 kb ladder, BRL) are positioned on the left sides

instruction. The concentration of total RNA was measured by NanoDrop 2000 spectrophotometer (Thermo Scientific, USA), while quality assessment for the integrity of RNA samples was confirmed by Agilent Bioanalyzer 2100 RNA Nano chip (Agilent, USA) using RNA 6000 Nano assay kit (Agilent, USA). The isolation and enrichment of
poly(A)-containing mRNA was performed from $5 \mu \mathrm{g}$ total RNA of each samples using Dynabeads mRNA Purification Kit (Life Technologies, USA) and further used for cDNA synthesis using cDNA Synthesis System (Roche, Basel, Switzerland) as per the manufacturer's protocol. The cDNA samples were sheared via nebulization into 
small fragments and were used for library construction using TruSeq RNA Library Preparation Kits. The resulting libraries were then paired-end sequenced (2x101bp) on an Illumina HiSeq2000 platform (Illumina) using myGenomics (Atlanta, USA) sequencing services. The raw sequencing data were subjected to removal of Illumina adapter sequences and quality filtering for empty reads and low-quality sequences (reads with unknown " $\mathrm{N}$ " sequences) using Trimmomatic v0.30 program [52]. The short reads below than 50 bases were dropped to exterminate the sequencing artifacts and the quality of reads was evaluated using FASTQC toolkit [53]. The high-quality reads were de novo assembled using CLC genomics workbench (v.10.0.1) with default parameters (mismatch cost $=2$; insertion cost $=3$; deletion cost $=3$ ) into unique transcript sequences, termed as unigenes. All assembled unigene sequences were queried against the hop transcriptome database of HopBase genomic resources repository (http:// hopbase.cgrb.oregonstate.edu/) using MEGABLAST at E-value $<1 \mathrm{e}^{-3}$, with a cutoff of percentage identity more than $95 \%$ and alignment length greater than $100 \mathrm{bp}$. The unigene sequences were also aligned to the hop draft genome assembly [54] using Spaln2 program [55]. The quantitative assessment and completeness of assembled unigenes was performed using BUSCO (Benchmarking Universal Single-Copy Orthologs; version 3.0) software [56].

\section{Functional annotation of the unigenes}

The assembled sequences were aligned against the NCBI non-redundant (nr) protein database using BLASTX at E-value cut-off of $1.0 \mathrm{E}-3$. Blast homology searches and homology-based functional annotations were performed using Blast2GO command line version 1.3.0 tool (https://www.blast2go.com/) [57]. Gene Ontology (GO; http://www.geneontology.org/) terms describing the biological process, molecular function, and cellular component were assigned to the unigenes through Blast2GO. The unigene sequences were also aligned to the Clusters of Orthologous Group (COG) database to predict and classify functions [58]. The single-directional best hit (SBH) method was used for KEGG (The Kyoto Encyclopedia of Genes and Genomes) pathway assignment of the assembled sequences using the online KEGG Automatic Annotation Server (KAAS; http:// www.genome.jp/kegg/kaas/) to gain an overview of the gene pathway networks [59]. The PlantTFcat online tool was used for the identification of unigenes encoding transcription factor [60].

\section{Identification of differentially expressed genes and their network and pathway analysis}

After assembly and annotation, the expression levels of each unigene between WT and WW-transformant lines were calculated by mapping clean read sets on the reference transcriptome as FPKM value (Fragment per kilobase of transcripts effective length per million fragments mapped to all transcripts) by expectation-maximization (RSEM) protocol using in-built scripts in the Trinity software package [61]. The normalization of the data among different libraries was performed using the Trimmed Mean of M-values normalization method in Trinity. The obtained count value was exported to Bioconductor software package DESeq2 [62] to identify the differentially expressed gene transcripts (DEG) using the Benjamini-Hochberg false discovery rate $(\mathrm{FDR})<0.05$. The expression of a particular sequence was considered significantly different when the adjusted $P$-value obtained using this method was $\leq 0.05$ and there was at least a two-fold change $(\geq 2$ or $\leq-2)$ in the sequence count between WT and WW-libraries. The FPKM values for each transcript were log-transformed and normalized, which was subsequently used for calculation of matrix distance with Euclidean distance and complete-linkage methods. The $\mathrm{R}$ statistics package heatmap3 [63] was used for construction of heatmap. The DEGs were used for GO terms/KEGG pathway enrichment analyses using hypergeometric test equivalent to one-tailed Fisher's exact test with a FDR value of 0.05 using the AgriGO toolkit [64], functional analysis and pathway visualization was performed using MapMan tool [65]. Protein families were assigned to DEGs by searching them against the Protein family (Pfam) database using HMM-based tool pfamscan [66].

\section{Validation using real-time quantitative RT-PCR}

The differential expression of eight structural and six regulatory genes associated with phenylpropanoids and flavonoids biosynthesis pathway was subjected for a real-time quantitative reverse transcriptase PCR (qRT-PCR) validation of the transcriptome data. The first strand cDNA was synthesized from $5 \mu \mathrm{g}$ of aliquots of the total RNA extracted for sequencing as described earlier using Superscript $^{\circ}$ III First-strand cDNA Synthesis system (Invitrogen, USA) according to the manufacturer's instructions. Each qRT-PCR mixture consisted of $200 \mathrm{ng}$ first strand cDNA, $10 \mu \mathrm{l}$ SYBR Green Real-Time PCR Master Mix (Invitrogen, USA), $10 \mathrm{pmoL}$ of forward and reverse gene-specific primers (Additional file 2: Table S1). Additionally, primers specific to hop GAPDH gene were used as an endogenous control to normalize the expression level of each gene [67]. PCR amplification was performed in an IQ5 Real-Time PCR Detection System (Bio-Rad, CA, USA) under the following conditions: $95^{\circ} \mathrm{C}$ for $10 \mathrm{~min}$, followed by 40 cycles at $95^{\circ} \mathrm{C}$ for $15 \mathrm{~s}$ and at $60^{\circ} \mathrm{C}$ for $1 \mathrm{~min}$. The melting curve analysis was performed to assess the specificity of the PCR primer specific product by maintaining the reaction at $95^{\circ} \mathrm{C}$ for $1 \mathrm{~min}$, cooling the sample to $55^{\circ} \mathrm{C}$ for $1 \mathrm{~min}$ and further heating to $95{ }^{\circ} \mathrm{C}$ at a rate of $0.5{ }^{\circ} \mathrm{C}$ per $6 \mathrm{~s}$. The relative 
expression levels (fold-change) of the selected genes were calculated using the comparative $\mathrm{Ct}\left(2^{-\Delta \Delta \mathrm{Ct}}\right)$ method [68]. For each sample, the experiment was carried out in three independent technical replicates and based on that error bars were calculated.

\section{Analysis of polyphenols and flavonoids content in WW-transgenic line}

The extract preparation (methanol: $\mathrm{H}_{2} \mathrm{O}$ ) from dried leaf samples of WT and WW-transgenic plants, HPLC analysis of total phenolics and flavonoids content were performed as described previously [17, 69].

\section{Results}

Molecular, biochemical and morphological analysis of WW-transgenic lines

For this study, we have selected three independent hygromycin resistant WW-transgenic lines (B11, B23, and B24) generated using Agrobacterium-mediated transformation methods. The WWpPCV91 plasmid-based plant expression vector harboring dual expression cassettes (Fig. 2a) was used to create these transgenic lines. Southern blot analysis using HlWDR1 and hygromycin phosphotransferase $(H P T)$ gene confirmed that T-DNA was stably inserted into chromosomal DNA (Fig. 2b). The qRT-PCR of the regenerated plantlets (3-month-old) confirmed the presence and overexpression of transgenes in WW-lines (Additional file 4: Figure S3). The plants were transferred to the greenhouse and monitored throughout their vegetative development phase (year: 2016-2017) with special emphasis on plant morphology and growth. The growth performance of WW-transgenic lines was observed to be superior to WT-plants with much larger immature leaves of comparable position on plants of the same age (Additional file 5: Table S2; Additional file 3: Figure S2D). The chlorophyll content in WW-transgenic plants was higher than WT, suggesting the better photosynthetic performance of WW-lines (Additional file 5: Table S2). The lupulin gland distribution in transgenic lines was almost similar to that WT with respect to the differences of the epidermal cell size.

\section{Sequencing and de novo transcriptome assembly analyses} To obtain a comprehensive overview of the gene expression pattern in WW-transgenic lines, RNAseq libraries were constructed from the leaf tissue from individual two independent transgenic (B11 and B23) and WT (Osvald $72 \mathrm{cv}$.) lines with their technical replicates. High throughput sequencing run generated over 29 and 32 million raw reads in WT and WW samples, respectively (Table 1). After the removal of the adaptor and filtering out the low-quality reads at high stringency using Trimmomatic software, over 24 and 26 million high-quality reads were obtained for WT and WW groups, respectively. The
Table 1 Statistics of RNA-seq analysis and assembly for hop

\begin{tabular}{lllc}
\hline Item & Library & Number & Total Bases (GB) \\
\hline Raw read & WT & $29,177,758$ & 2.20 \\
& WW & $32,818,870$ & 2.50 \\
Clean read & WT & $24,399,800$ & 1.79 \\
& WW & $26,889,520$ & 1.96 \\
Average Length (bp) & WT & 422 & \\
& WW & 416 & \\
Unigenes & & & \\
No. of Unigenes (n) & & 23,666 & \\
Average Length (bp) & & 436 & \\
Maximum Length (bp) & & 2200 & \\
Minimum Length (bp) & & 106 & \\
\hline
\end{tabular}

transcriptome datasets (raw data) generated in this study have been deposited at the Sequence Read Achieve (SRA), National Centre for Biotechnology Information (NCBI) with the accession numbers SRR6308266, SRR6308267, SRR6308195 and SRR6308265 as biological replicates for WT and WW, respectively. All clean reads were subjected to the de novo assembly, which resulted in a total of 23,666 unigenes with size ranging from $157 \mathrm{bp}$ to $2002 \mathrm{bp}$ with an average unigene size of 436 bp (Table 1). The average unigene size was much longer (436 bp) than those identified in previous studies in Withania somnifera (200 bp) [70], Ipomoea batatas (202 bp) [71], Eucalyptus grandis (247 bp) [72] and Physalis peruviana (371 bp) [73]. The N50 of 452 bp value was obtained for the current transcriptome assembly and 9962 unigenes (42.10\%) were longer than 400 bp (Fig. 3a). The average GC content of hop unigenes was $41.10 \%$, which was comparable to the GC levels of unigenes of Sophora flavescens (39.9\%) [74], chickpea (40.3\%) [75], Spinacia oleracea (42.5\%) [76] and Glycine $\max$ (43\%) [77]. The comparative analysis of unigene sequences to hop transcriptome database available in HopBase showed that a total of 21,545 (91.03\%) matched with greater than 95\% sequence identity, indicated the broad representation of our unigenes. Approximately 18,565 (78.45\%) unigenes were mapped to the draft hop genome assembly, which could be due to the incompleteness of the genome sequence, level of genetic variation between cultivars and the existence of large amounts of intergenic noncoding RNAs etc. [78], suggesting that our unigenes could serve as a valuable complementary resource for hop genomics.

\section{Functional annotation and classification of hop transcriptome sequences}

Functional annotation of the assembled sequences against the NCBI non-redundant (nr) protein database showed that $18,048(76.26 \%)$ matched to the $\mathrm{nr}$ protein database, whereas 5618 (23.74\%) did not exhibit significant homology 


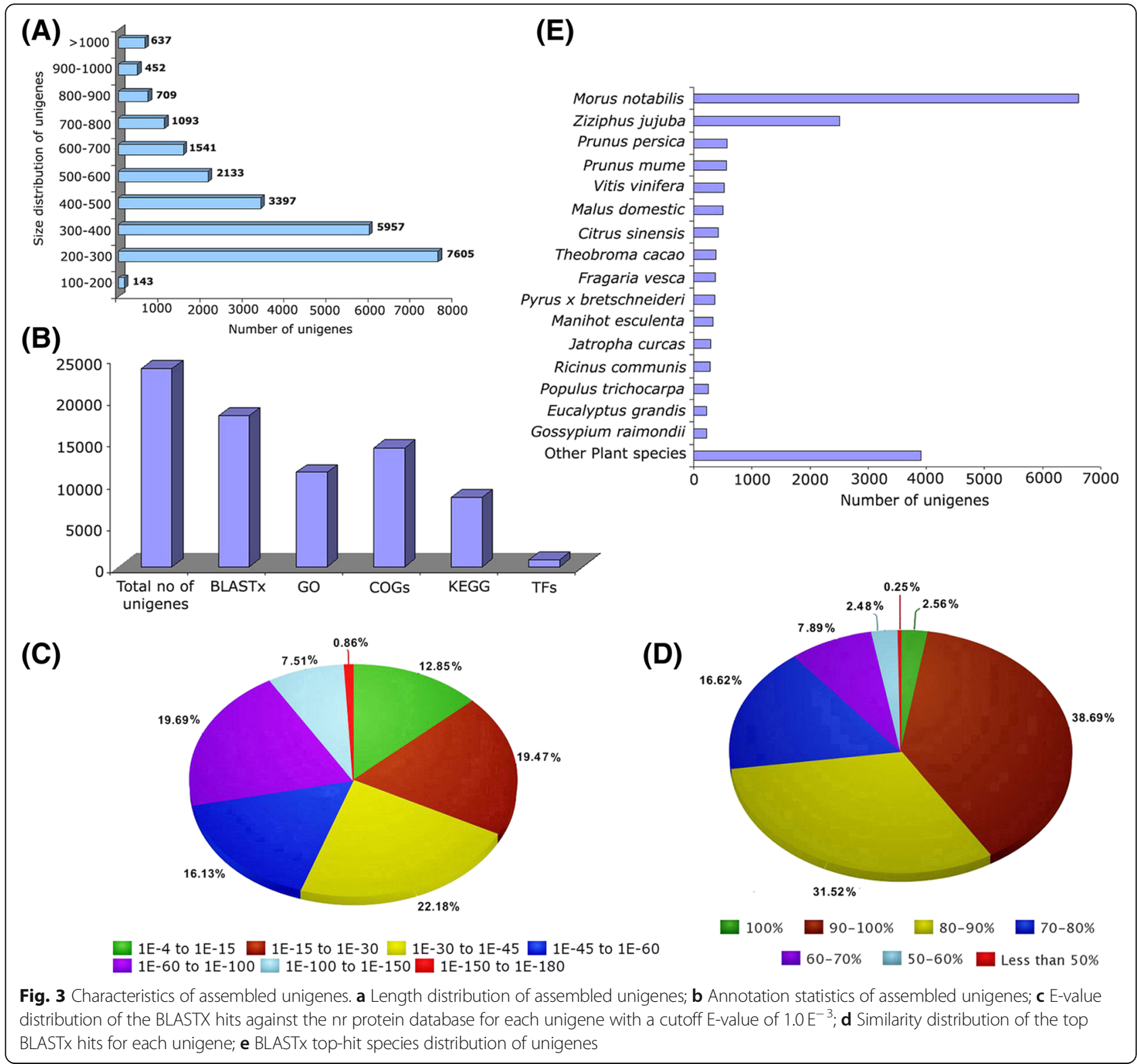

with sequences in the $\mathrm{nr}$ database (Additional file 6: Table S3, Fig. 3b). The E-value distribution of aligned unigenes against $\mathrm{nr}$ database showed that $60.90 \%$ of unigenes had an E-value of less than $1.0 \mathrm{E}^{-50}$ (Fig. 3c). The similarity distribution of the aligned unigenes compare to sequences in the $\mathrm{nr}$ database illustrated that $41.25 \%$ of unigenes had significant homology higher than $90 \%$, followed by $58.51 \%$ of the sequences with homology between 50 and $90 \%$; whereas only $0.25 \%$ of the sequences had homology lower than $50 \%$ (Fig. 3d). Furthermore, the species distribution analysis of unigenes based on their BLASTx alignment against the $\mathrm{nr}$ protein database showed that approximately $47.82 \%$ of total unigenes were matched with sequences from six dicotyledonous species, namely, Morus notabilis (27.96\%), Ziziphus jujuba (10.61\%), Prunus persica (2.46\%), P. mume (2.41\%),
Vitis vinifera (2.21\%) and Malus domestica (2.15\%) (Fig. 3e). The annotation information for the majority of unigenes based on their homologous matches was obtained, which demonstrates the high accuracy of assembled transcript sequences. BUSCO analysis against a core set of 1440 single-copy orthologous genes of plants indicated the presence of $62 \%$ as complete, $26 \%$ fragmented, and $12 \%$ missing orthologs in our de novo assembly. The functional classification of unigenes based on BLAST search against the gene products in the GO database classified 14,184 matched unigenes into the three main GO categories, including 40 functional groups (Fig. 3b). A total of 72,581 GO functional terms was obtained, among them the biological process comprised the major category $(35,254,48.57 \%)$ followed by cellular component $(22,022,30.34 \%)$ and molecular functions $(15,305$, 
21.09\%) (Additional file 7: Figure S4A). Consistent with our observations, the prominence of the biological process over the molecular function and cellular components under the GO categories have been reported in other plant species such as cucumber [35], sesame [79], wheat [80], spinach [76] and many more.

It was found that 16,386 of the total unigenes have significant homology in the COG database (Fig. 3b) with multiple functions resulting in 74,723 functional annotations and these unigenes were classified into 25 categories (Additional file 8: Figure S5A). Of these categories, "general functional prediction only" (12,123, 16.22\%) represented the largest group, followed by "inorganic ion transport and secretion" (10,128, 13.55\%), "amino acid metabolism and transport" (7136, 9.55\%), "post-translational modification, protein turnover, chaperone function" (6367, 8.52\%), whereas the smallest groups were assigned to "nuclear structure" (53, 0.07\%), followed by "cell motility" (112, 0.15\%) and "extracellular structures" (134, 0.18\%) (Additional file 8: Figure S5A).

To further investigate the biological functions of putative proteins and their biochemical pathways, the 8271 KEGG annotated unigenes (Fig. $3 \mathrm{~b}$ ) were grouped into five different functional groups (Table 2). KEGG pathway analysis annotated largest number of unigenes against "metabolism" with most of them represented "carbohydrate metabolism" (5.78\%), "amino acid metabolism" (4.33\%), "lipid metabolism" (3.64\%), "energy metabolism" (3.54\%), "biosynthesis of secondary metabolites" (1.73\%) and other sub-categories. Strikingly, classified unigenes into the category "biosynthesis of secondary metabolites" were found to be linked with various secondary metabolites associated pathways, such as sesquiterpenoid and triterpenoid biosynthesis, flavonoid biosynthesis, and prenylflavonoids biosynthesis and many more. This observation was consistent with the previous notion that most of the secondary metabolite biosynthesis related genes are expressed in hop leaf tissues at detectable levels [11]. A total of 4781 unigenes were annotated into pathways related to "genetic information processing" included genes involved in transcription, translation, replication and repair, and protein folding, processing, and degradation. In addition, unigenes were also classified into pathways related to "cellular processes" and "environmental information processing" which accounted for 1676 and 1565 unigenes respectively of the KEGG annotated sequences (Table 2).

A total of 840 unigenes, accounted for $3.55 \%$ of the transcriptome, were classified into 72 putative transcription factors families (Additional file 9: Table S4). Among

Table 2 Classification statistics for unigenes (UG) and differentially expressed genes [up-regulated (UR) and down-regulated genes (DR)] in WW-transformant lines according to KEGG pathway analysis

\begin{tabular}{|c|c|c|c|c|c|c|c|}
\hline \multirow[t]{2}{*}{ KEGG categories } & \multicolumn{3}{|c|}{ Number of } & \multirow[t]{2}{*}{ KEGG categories } & \multicolumn{3}{|c|}{ Number of } \\
\hline & $\overline{U G}$ & UR & $\mathrm{DR}$ & & $\overline{U G}$ & UR & $\overline{D R}$ \\
\hline \multicolumn{8}{|l|}{ Metabolism } \\
\hline Carbohydrate Metabolism & 772 & 20 & 3 & Cellular Process & & & \\
\hline Energy metabolism & 473 & 6 & 6 & Transport and catabolism & 1011 & 11 & 8 \\
\hline Lipid metabolism & 487 & 24 & 2 & Cell growth and death & 331 & 7 & 1 \\
\hline Nucleotide metabolism & 210 & 1 & 3 & Cellular community - eukaryotes & 99 & 0 & 0 \\
\hline Amino acid metabolism & 578 & 4 & 2 & Cellular community - prokaryotes & 83 & 4 & 0 \\
\hline Metabolism of other amino acids & 221 & 9 & 4 & Cell motility & 152 & 2 & 0 \\
\hline Glycan biosynthesis and metabolism & 349 & 5 & 1 & Environmental information processing & & & \\
\hline Metabolism of cofactors and vitamins & 325 & 5 & 6 & Membrane transport & 599 & 19 & 4 \\
\hline Metabolism of terpenoids and polyketides & 181 & 5 & 1 & Signal transduction & 779 & 18 & 4 \\
\hline Biosynthesis of other secondary metabolites & 231 & 10 & 4 & Signaling molecules and interaction & 187 & 6 & 0 \\
\hline Xenobiotics biodegradation and metabolism & 127 & 3 & 2 & Unclassified & & & \\
\hline Enzyme families & 921 & 9 & 7 & Metabolism & 229 & 12 & 3 \\
\hline Genetic information processing & & & & Genetic information processing & 68 & 0 & 0 \\
\hline Transcription & 744 & 5 & 3 & Cellular processes and signaling & 104 & 2 & 0 \\
\hline Translation & 1574 & 10 & 12 & Viral protein family & 0 & 0 & 0 \\
\hline Folding, sorting and degradation & 1703 & 9 & 10 & Poorly characterized & 69 & 0 & 1 \\
\hline Replication and repair & 760 & 7 & 4 & & & & \\
\hline \multirow[t]{2}{*}{ RNA family } & 0 & 0 & 0 & & & & \\
\hline & & & & Total & 13,367 & 213 & 91 \\
\hline
\end{tabular}


the 72 transcription factors families, $\mathrm{C} 2 \mathrm{H} 2$, WD40-like, Hap3/NF-YB, CCHC(Zn), GRAS, MYB-HB-like, WRKY, bHLH, AP2-EREBP, SET, bZIP, PHD families were the top 12 classes (Additional file 10: Figure S6).

\section{Identification of differentially expressed genes and functional analysis}

To compare the gene expression levels in the WT and WW-libraries, FPKM values of assembled unigenes were calculated. The mapping of all the reads onto the non-redundant set of hop transcripts revealed that the number of reads corresponding to each transcript ranged from 0.04 to 1972.80 for WT (FPKM) and from 0.09 to 4896.07 (FPKM) for WW library, respectively, indicating a very wide range of expression levels of hop transcripts (Additional file 11: Table S5). Transcriptome comparison resulted in the identification of 522 differentially expressed genes (DEGs, $p \leq 0.05, \log \mathrm{FC} \geq 2$ or $\leq-2$ ), among them 385 were found to be significantly up-regulated whereas, 137 were significantly down-regulated in WW-transgenic line compared to WT hop plant. Approximately $89 \%$ of DEGs (349 up-regulated genes and 115 down-regulated) were annotated against the nr protein database of NCBI (Additional file 11: Table S5).

Hierarchical cluster analysis based on FPKM values arranged 522 DEGs into twelve major clusters and were consistent within WT and WW-lines of hop (Fig. 4). Cluster I and II were enriched with unigenes encoded enzymes and transcription factors involved in the secondary metabolite synthesis, namely PAL, C4H, 4CL, CHS_H1, PRT1, OMT1 VPS, flavanone 3-dioxygenase $(F 3 D)$, Isoflavone 2'-hydroxylase $(I 2 H)$, HlWRKY1, HlWDR1, HlMyb2, $H l \mathrm{Myb} 3, H l \mathrm{bHLH} 2$. The genes grouped in cluster VIII were found to be down-regulated in WW transformants. Taken together, heat map results again reinforce the elevated level of expression of genes associated PF and BA biosynthesis pathway in WW transformants.

Functional categorization based on GO enrichment analysis using the Fisher's exact test at the false discovery rate of 0.05 provided the statistically significant GO terms for DEGs. The GO annotation of DEGs categorized 424 unigenes into 30 functional groups, whereas, 98 unigenes were not classified (Additional file 7: Figure S4B). Among GO categories, "biological process" comprising the major domain of DEGs followed by "cellular component" and "molecular function". Among the various biological process categories, functional enrichment analysis of DEGs revealed that genes involved in "cellular process" and "metabolic process" were significantly enriched (Table 3) and up-regulated (Additional file 7: Figure S4B). Strikingly, unigenes involved in growth, developmental process, and anatomical structure morphogenesis were found to be enriched suggested that some aspects related to these processes might have been activated in WW-transformant lines. The improved growth performance and larger leaf size of WW-transformant lines in optimal growth condition (Additional file 3: Figure S2D) could be correlated with these observations. In addition, GO terms related to "binding", "catalytic activity", "hydrolase activity", "transferase activity", "transporter activity" and "transcription factor activity" were enriched among "molecular function", whereas within "cellular components" category "cell", "cytoplasm", "membrane", "plastids" domains were enriched (Table 3). This observation suggested that these functions were enhanced in WW-transformant lines. Analysis of the COG classification of DEGs indicated their grouping in all COG functional categories except $\mathrm{N}$ (cell motility) category (Additional file 8: Figure S5B). The identified DEGs were subjected to KEGG pathway enrichment analysis. A total of $162(42.08 \%)$ up-regulated and 67 (48.91\%) down-regulated unigenes were annotated and assigned to 7 main categories (Table 2).

Furthermore, DEGs were imported into MapMan for pathway-based analysis and visualization to gain an unbiased overview of important pathways or biological processes changed in WW-transformants. Consistent with GO analysis, DEGs associated with the secondary metabolite biosynthesis pathway showed upregulation (Fig. 5) in WW-transformants. Moreover, genes involved in cell wall metabolism, lipid metabolism, light reactions, and photorespiration were up-regulated, while those involved in starch degradation were down-regulated.

\section{Validation of secondary metabolite biosynthetic pathway associated DEGs by qRT-PCR}

Based on the result of Illumina sequencing data, the expression profiles of selective structural and regulatory genes involved in PF, BA and flavonol biosynthesis pathways were analyzed in leaves of WT and WW-transgenic hop lines using qRT-PCR. The real-time gene expression analysis suggested up-regulation of structural genes, namely PAL, C4H, 4CL, CHS_H1, PRT1, OMT1, VPS and genes encoding regulatory proteins, namely HIWRKY1, HlWDR1, HlMyb2, HlMyb3, HlbHLH2 (Fig. 6). The expression level of $H l \mathrm{WRKY} 1$ and $H l \mathrm{WDR} 1$ was comparable in all WW-transgenic lines. As expected, the expression level of HlWRKY1 was several folds higher than HlWDR1, reflected its auto-activation activity [19]. The expression of the gene encoding $H l$ Myb1 regulatory protein was not increased, which was similar to the transcriptome data analysis. Among the structural genes involved in PF and BA biosynthesis pathways, the maximum enhancement in expression was observed in the case of $\mathrm{CHS}$ _H1. However, the distinct expression level of other structural genes associated with flavonol and anthocyanin pigmentation pathways was not observed, except for the $F 3 H$ gene. The expression patterns of all the selected genes analyzed by 


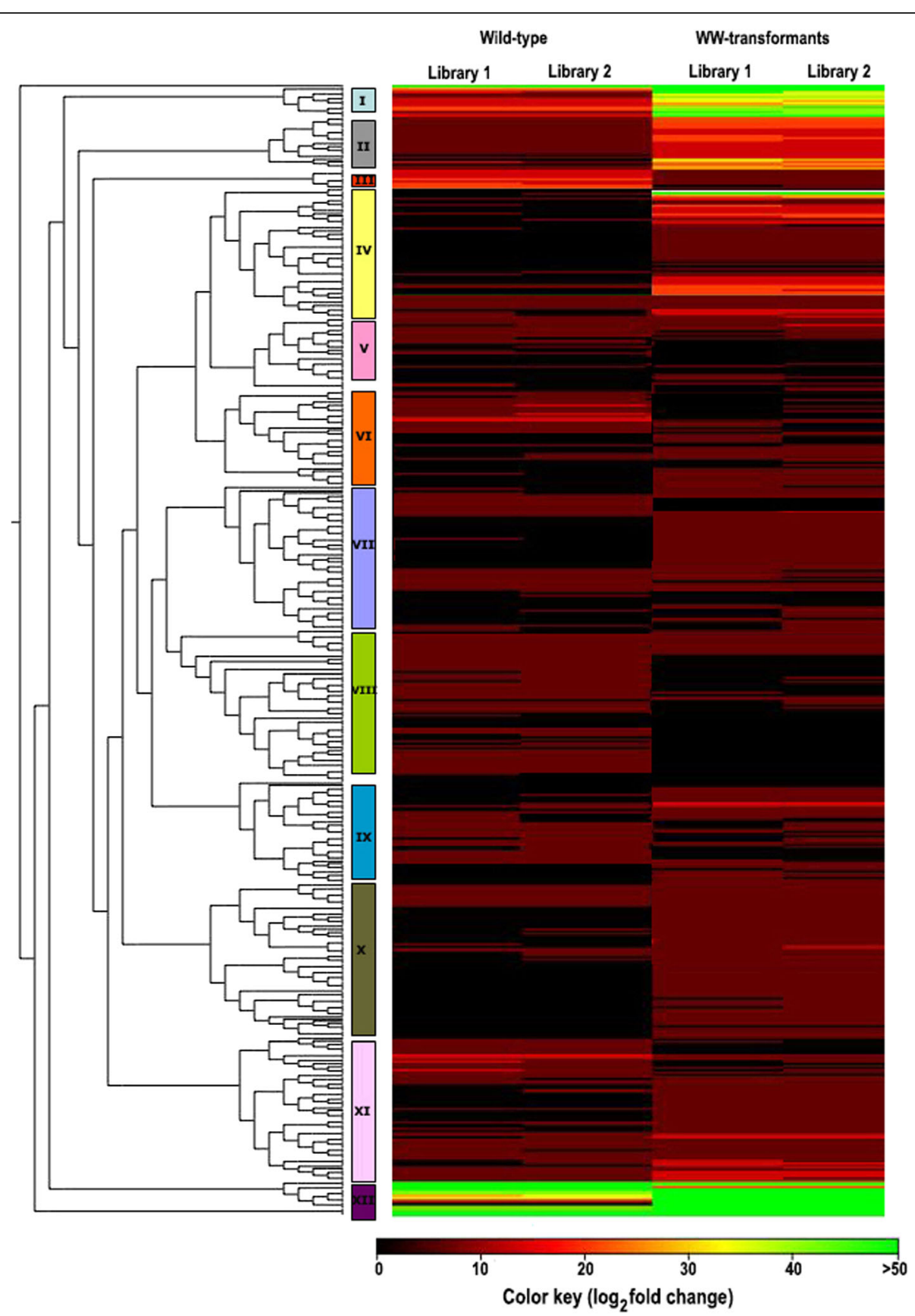

Fig. 4 Heat map and complete linkage hierarchical clustering of differentially expressed genes in leaves of WW transformants compared with wild-type hop. Colors on vertical represent the clustered genes based on gene expression, the horizontal line represents the single gene and color of the line indicates the average gene expression in WW transformants. The signal ratios were shown in a black-green color scale, where green indicated high expression level and black indicated low expression level

qRT-PCR were consistent with the DEGs analysis (Fig. 6; Additional file 11: Table S5).

\section{Analysis of flavonoids content in WW-transgenic line}

Hop leaves contain very low content of PF and BA compared to mature cones [81] and therefore, their quantification is difficult to analyze using HPLC method [82].
However, in order to evaluate the possible change in secondary metabolite content in leaves of WW-transgenic lines, we performed metabolite fingerprinting by HPLC. The contents of $\alpha$-bitter acids $(\sim 60$ fold) and xanthohumol ( 40 fold) were enhanced significantly (Additional file 12: Figure S7) in leaves of WW-transgenic lines compared to WT hop. 
Table 3 Gene Ontology (GO) Functional Enrichment Analysis of differentially expressed genes in hop

\begin{tabular}{|c|c|c|c|c|c|c|}
\hline GO ID & Ontology & Category & Number of DEGs in subgroup & Number of unigenes in subgroup & $P$-value & FDR \\
\hline GO:0008152 & metabolic process & $P$ & 161 & 10,614 & $8.30 \mathrm{E}-21$ & $6.80 \mathrm{E}-19$ \\
\hline GO:0009058 & biosynthetic process & $P$ & 62 & 5118 & 0.00039 & 0.008 \\
\hline GO:0009987 & cellular process & $P$ & 32 & 11,684 & $9.50 \mathrm{E}-07$ & 3.20E-05 \\
\hline GO:0050896 & response to stimulus & $P$ & 32 & 4057 & 0.53 & 1 \\
\hline GO:0006950 & response to stress & $P$ & 24 & 2320 & 0.11 & 0.82 \\
\hline GO:0032502 & developmental process & $P$ & 22 & 2304 & 0.21 & 1 \\
\hline GO:0048856 & anatomical structure development & P & 10 & 1726 & 0.88 & 1 \\
\hline GO:0003824 & catalytic activity & $\mathrm{F}$ & 176 & 9638 & 4.60E-34 & $2.30 \mathrm{E}-32$ \\
\hline GO:0005488 & binding & $\mathrm{F}$ & 112 & 11,258 & 0.0025 & 0.031 \\
\hline GO:0016787 & hydrolase activity & $\mathrm{F}$ & 74 & 3478 & $2.90 \mathrm{E}-15$ & $7.20 \mathrm{E}-14$ \\
\hline GO:0016740 & transferase activity & $\mathrm{F}$ & 55 & 3321 & $1.50 \mathrm{E}-07$ & $2.50 \mathrm{E}-06$ \\
\hline GO:0005215 & transporter activity & $\mathrm{F}$ & 17 & 1473 & 0.081 & 0.5 \\
\hline GO:0003700 & transcription factor activity & $\mathrm{F}$ & 8 & 2173 & 1 & 1 \\
\hline GO:0005623 & cell & C & 129 & 15,217 & 0.16 & 1 \\
\hline GO:0016020 & membrane & C & 71 & 4068 & 1.40E-10 & $6.40 \mathrm{E}-09$ \\
\hline GO:0005737 & cytoplasm & C & 48 & 6822 & 0.83 & 1 \\
\hline GO:0009536 & plastids & C & 15 & 2965 & 0.98 & 1 \\
\hline
\end{tabular}

$P$ Biological process, $F$ Molecular function, $C$ Cellular component

\section{Discussion}

Hop plants have been widely used in the brewing industry and practiced as traditional medicine since ancient times. Since hop extracts have beneficial and positive effects on human health and exhibit a wide range of biological activities with several other potential interesting applications, therefore, it has been the subject of systematic studies for more than three decades. The recent application utilizes crude spent hops extract as a botanical dietary supplement [83] and as an antifeedant activity to suppress stored-product insect pest populations [84]. For these reasons, conventional breeding and genetic engineering programs have been implemented in hop plant in efforts to enhance levels of biologically active compounds of interest $[23,85,86]$. The transcription factors have emerged as a promising candidate for genetic engineering owing to their role as master regulators of multiple target genes [26, 87]. In this context, our earlier study pertaining to $H I M Y B 3$ overexpression in hop demonstrated several folds enhanced accumulation of flavonoids and phloroglucinols accompanied by enhanced expression of CHS_H1, CHI, F3'H, VPS and OMT1 genes [25]. Similarly, the heterologous expression of Arabidopsis transcription factor (AtMYB12) in tomato resulted in significant enhancement of flavonols and phenolics content in fruits by activating genes involved in the flavonoid biosynthesis pathway [28].

The HIWRKY1 transcription factor shares significant homology to Arabidopsis AtWRKY75 [19], which regulates phosphate acquisition and modulates root architecture
[21], whereas $H /$ WDR1 transcription factor shows homology to Transparent Testa Glabra 1 (TTG1), encoding WD40 repeat transcription factor, which is involved in many aspects of plant development, regulation of flavonoid/anthocyanin biosynthesis, accumulation of seed storage reserves and trichome formation in leaves [22]. In this study, HIWRKY1 and HIWDR1 transcription factors were constitutively over-expressed in hop transgenic lines to enhance PF and BA content. Furthermore, we investigated for genome-wide alteration of steady-state transcript levels by comprehensive transcriptome profiling to build up information about the overall impact of constitutive expression of $H l \mathrm{WRKY} 1$ and $H l \mathrm{WDR} 1$ transcription factors over the morphological, developmental attributes and other related pathways of hop.

Transcript profiling and comparative transcriptome analysis with the aid of the high-throughput mRNA sequencing (RNA-Seq) technologies have frequently been used to identify networks of differentially expressed genes and genes expression patterns in several plant species. In this study, high-throughput sequencing generated more than 51 million high-quality reads, which were assembled into 23,666 unigenes. Subsequently, unigenes were functionally annotated using nr, GO, COGs and KEGG databases. The annotation results provide a valuable resource for further investigating the specific processes, pathways, and functions in the hop. Approximately $24 \%$ of unigenes were not annotated and could be considered as novel transcripts or alternative splice variants. The results of DGEs analysis suggested the 


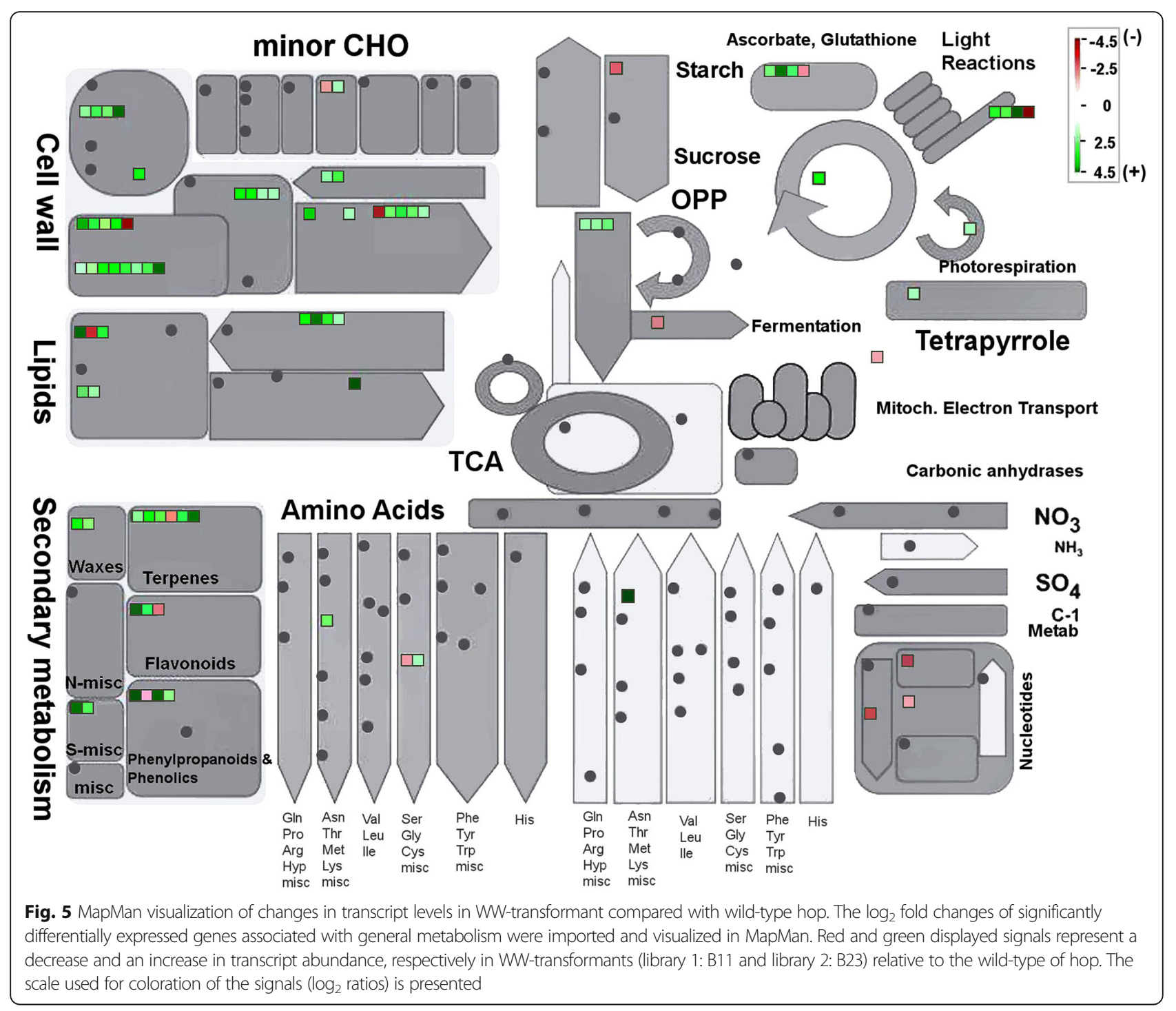

alteration in gene expression level in WW-transformants and these genes were not only the components of $\mathrm{PF}$ and $\mathrm{BA}$ biosynthesis pathways but also belonging to other pathways involved in carbohydrate metabolism and lipid metabolism. Such modulation facilitates the enhancement of precursor and substrate molecules for flavonoid biosynthesis via central metabolic pathways connecting malonyl-CoA (product of carbon metabolism) and coenzyme A esters (product of lipid metabolism) with the plant flavonoid biosynthesis [88]. The up-regulation of PAL gene in WW-transformants line also suggested the enhanced flux of substrate towards the flavonoid pathway. The substrate channeling is a common event in cellular metabolism and such type of metabolic reprogramming involves a dramatic biosynthesis of transporters, which play a pivotal role in the efficient channeling of substrates ranging from organelles to the whole plant [89]. Nevertheless, we cannot rule out this possibility as genes encoding sugar, amino acid, lipid and ammonium transporters were differentially regulated in WW-transgenic hop plants. In this context, the integration of metabolomics, transcriptomics and proteomics data could be used as a future prospect to understand interactions between metabolites and genes/ proteins and their relations in substrate channeling in flavonoid biosynthesis. Genes involved in photosynthesis (chlorophyll a/b binding proteins, chloroplastic protein, etc.) and stress response (pathogenesis-related protein: PR-1; PR-10, ethylene-responsive transcription factor 1B, TMV resistance protein N-like, $\mathrm{ABC}$ transporters etc.) were up-regulated. Several plant species exploit flavonoids as signaling molecules and their enhanced expression concurrently activates the expression of stress-related genes $[30,90,91]$. These observations indicate that increased flavonoids accumulation probably triggered the signaling cascades leading to the activation of stress-related genes 


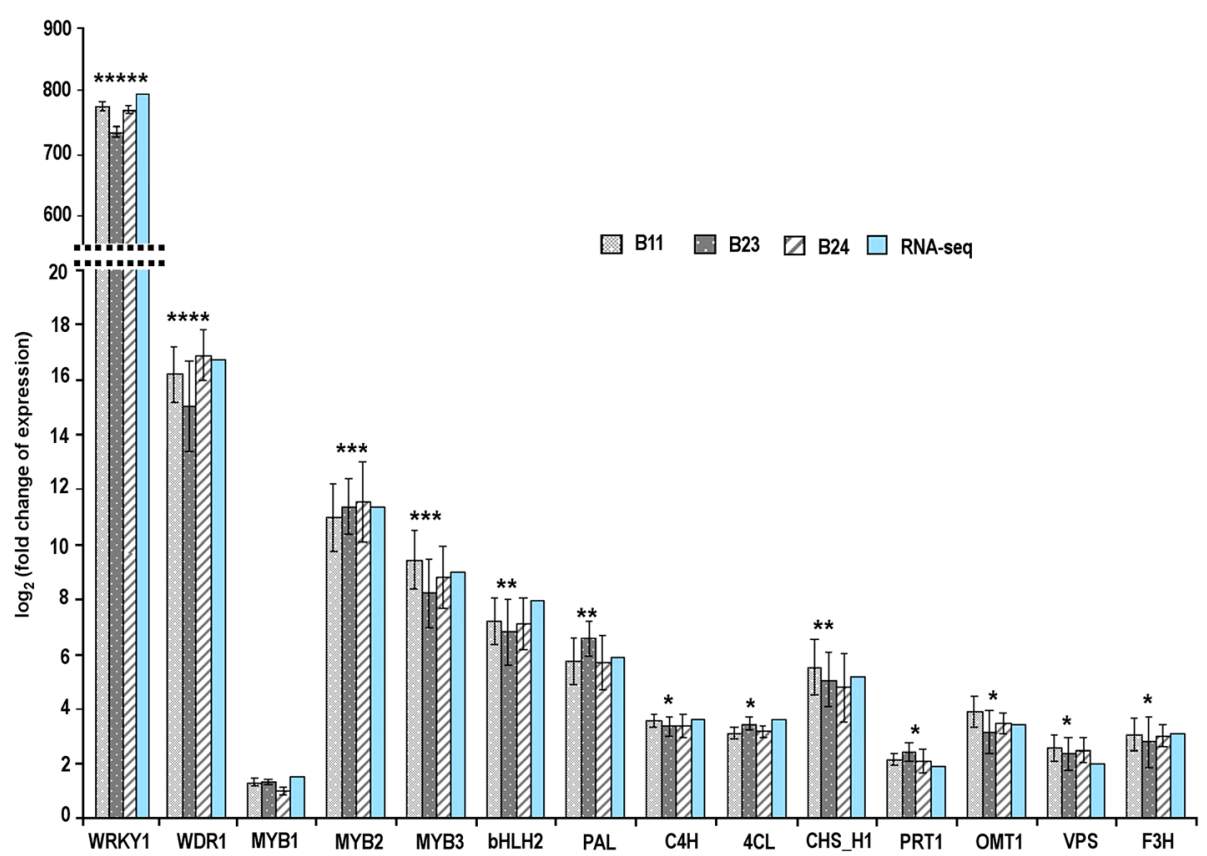

Fig. 6 Validation of RNA sequencing by RT-qPCR. Graph showing fold change of the structural and regulatory genes in a leaf of hop among transgenic lines (B11, B22, and B23) overexpressing HNRKY1 and HMDR1 transcription factors. PAL: phenylalanine ammonia lyase; C4H: cinnamate 4-hydroxylase, 4CL: coumarate coenzyme A ligase, CHS_H1: chalcone synthase isoform 1, PRT1: prenyltransferase 1; OMT1: O-methyltransferases isoform 1; VPS: valerophenone synthase, F3H: flavanone 3-hydroxylase. qRT-PCR analyses were normalized using GAPDH as an internal control gene. The fold change of each gene was calculated by the $2^{-\Delta \triangle C T}$ method. *Statistically significant differences $(P<0.05)$; **significant at $p<0.01$

in WW-transformants. The role of mitogen-activated protein kinase (MAPK) cascades via chromatin reprogramming by histone deacetylase 2 (HDAC2) in various stress response has been well documented in plants [92, 93], and therefore it is possible that modulation of MAPK cascades in WW transformants could cause the synergistic changes in HDAC2, which in turn through the chromatin reprogramming modulate the expression of genes associated with stress responses.

Several genes functioning in hormone biosynthesis and signaling pathways were differentially regulated in WW-transformants. In plants, auxin is an important growth hormone which regulates a wide array of growth and development processes [94]. The gene expression of the auxin-induced protein (X10a), IAA-amino acid hydrolase (ILR1) and phytohormone signaling component such as auxin-binding protein (ABP19a-like), were found to be up-regulated in WW-transformants and could be attributed to their better growth performance compared to WT-hop plants. The enhancement of secondary metabolites production through the process of metabolic reprogramming can modulate the expression level of various genes associated with phytohormone biosynthesis and signaling [95] and thus corroborate a plausible mechanism of modulation of genes associated with auxin and gibberellin acid biosynthesis and signaling pathway in WW transformants.
Among various modulators, light has been reported to be one of the most important environmental factors affecting flavonoid biosynthesis [96, 97]. Cryptochromes are blue, green and UV-A light flavoproteins photoreceptors and involved in photomorphogenesis, adaptive and growth processes, including biosynthesis of secondary metabolites, such as flavonoids in plants [98, 99]. Notably, in our RNA-sequencing data, cryptochromes interacting transcription factor TCP2 were up-regulated, suggesting that flavonoids levels appear to be sensitive to change prominently under the light in WW-transformants compared to WT hop. Similarly, the genes involved in flavonoid biosynthesis have been reported to be positively regulated by light in transgenic tobacco overexpressing AtMYB111 transcription factor [29]. In our experiments, HlWRKY1 expression level responded non-synergistically with that of $H l W D R 1$. The WRKY gene promoter consists of high frequencies of W-box cis-elements (WRKY binding site) and can autoactivate their own expression [100]. This fact explains the discrepancies associated with several fold higher transgene expression level HIWRKY1 compared to HIWDR1 and corroborated by our previous report relating the high frequency of the W-box motif on HIWRKY1 promoter [101] and a state of high expression of $H I$ WRKY1 sustained by autoactivation [19]. The important finding of the present study is that WW-transgenic lines exhibited up-regulation of all the structural genes, namely PAL, C4H, 4CL, 
CHS_H1, PRT1, OMT1 and transcription factors $H l \mathrm{Myb} 2 /$ $H l \mathrm{Myb} 3, H l \mathrm{bHLH} 2, H l \mathrm{WDR} 1$ of MBW complex involved in PF and BA biosynthesis pathway. The up-regulation of structural genes involved in terminal steps of PF and BA biosynthesis was in accordance with our previous reports that $H I$ WRKY1 and $H l W D R 1$ transcription factor through independent or combinatorial activity drives the direct activation of OMT1 and PRT1 genes, and with the interaction with transcription factors of MBW complex drives the indirect activation of CHS_H1 gene of PF and BA biosynthesis pathways [19]. However, interestingly, we observed the relative up-regulated expression level of the structural genes $P A L, C 4 H, 4 C L$ of the general phenylpropanoid pathway. Growing evidence suggests that the metabolite levels can regulate metabolic enzymes on various levels, from specific allosteric modulation to more complex transcriptional regulation [102]. Therefore, it is probable that enhanced substrate flux regulates the expression level of the PAL. In this study, novel transcription factors belonging to MYB, bHLH, WRKY families were found to be upregulated. The family members of these transcription factors are involved in the regulation of various biological processes, including signal transduction, secondary metabolism, development and stress responses $[103,104]$. The changes in expression level of new MYB, bHLH, WRKY transcription factors either corroborated their regulatory role in early step gene activation of PF and BA biosynthesis pathway or a role in the development and stress responses, which are needed to ascertain and unravel through further experimentations. Furthermore, the expression analysis revealed the upregulation of flavanone 3-hydroxylase $(F 3 H)$ gene without the modulation of genes involved in anthocyanin pigmentation and flavonol biosynthesis pathway. It seems that overexpression of HIWRKY1 and HIWDR1 may divert the enhanced flux of the common substrate (L-phenylalanine) more specifically towards PF and BA biosynthesis pathways compared to flavonols and anthocyanins biosynthesis pathways.

In this study, we conclude that $H l \mathrm{WRKY} 1$ and $H l \mathrm{WDR} 1$ overexpression lead to dramatic changes in genome-wide transcriptome with positive feed back impact on the expression levels of genes involved in PF and BA biosynthesis pathways. Our comprehensive study represents a valuable contribution towards strategies to analyze plausible changes in WW-transgenic lines and provides a novel contender gene for enhancing the PF and BA content in hop through genetic engineering or breeding program. The present study was conducted under greenhouse conditions during 2 years growing seasons of WW-transformant lines and WT hop plants. At the end of second year growing seasons, WW-transformant lines and WT hop plants have been reached a standard height but with inadequate flower initiation. In the coming year, WW-transformant lines and WT plants will be allowed to grow under contained field conditions to achieve typical flowering with maximum cone size and yield. Our future research aims at metabolite profiling of flavonoids, bitter acids, terpenes, fatty acids, sugars and differential expression analysis of genes using lupulin gland samples derived from the female cone of WW-transformants and WT hop plants to correlate the present results and evaluate the performance and accumulation of secondary metabolites in genetically modified hop in field-testing condition.

\section{Conclusion}

In the present study, we conclude that overexpression of $H l \mathrm{WRKY} 1$ and HlWDR1 leads to up-regulation of structural and regulatory genes involved in prenylflavonoid and bitter acid biosynthetic pathways without any deleterious effect on the hop plants. Since several metabolic pathways are interconnected in order to allow an adequate regulation, thus possibly the perturbation of $\mathrm{PF}$ and BA biosynthesis pathways could lead to dramatic changes in the genome-wide transcriptome. With the availability of cones of WW-transformants, the detailed RNA sequencing and metabolite profiling will be performed to correlate and strengthen our present findings that $H l \mathrm{WRKY} 1$ and $H l \mathrm{WDR} 1$ genes could be served as potential contender genes to be used for secondary metabolome improvement programmes.

\section{Additional files}

Additional file 1: Figure S1. Stereomicroscopic photograph showing the distribution of lupulin glands on the bracteole surface of a cone (A) and adaxial side of leaf surface (B) of Osvald's cultivar of hop. (JPG 173 $\mathrm{kb})$

Additional file 2: Table S1. Primers used for cloning into plant vector, probes preparation, and qRT-PCR analyses. (DOC $62 \mathrm{~kb}$ )

Additional file 3: Figure S2. Regeneration of WW-transgenic plants of hop via Agrobacterium-mediated transformation of nodal explants (A), Phenotypic comparison of the growth of in vivo-grown WW-transformant and wild-type hop plantlets (B), Representative 2-year-old WW-transgenic and wild-type hop plant growing in the greenhouse condition (C), Leaf morphology of 2-year-old WW-transformant compared to wild-type hop plants (D) (Scale: $5 \mathrm{~cm}$ ). (JPG $414 \mathrm{~kb}$ )

Additional file 4: Figure S3. Relative levels of transcription factor transgenes HIWRKY1, HIWDR1 expression in the leaves of three independent lines of hop transformed with HMRKY1 and HMDR1 genes using vector WWpPCV91. RT-qPCR analyses were normalized using GAPDH as a house-keeping gene. The fold change of each gene was calculated by the $2^{-\Delta \Delta C T}$ method. *Statistically significant differences $(P<$ 0.05); ${ }^{* *}$ significant at $p<0.01$. (JPG $109 \mathrm{~kb}$ )

Additional file 5: Table S2. Morphological and physiological characteristics of the WW-transgenic plants compared to a wild-type plant of hop. (DOC $32 \mathrm{~kb}$ )

Additional file 6: Table S3. unigenes with significant BLAST X hits against the $\mathrm{nr}$ protein database. (XLS $2444 \mathrm{~kb}$ )

Additional file 7: Figure S4. Gene Ontology (GO) classifications of assembled unigenes $(\mathbf{A})$ and differentially expressed genes in WW transformants compared with wild-type (B). The results are summarized in three main categories: Biological process, Cellular component and Molecular function. (JPG $468 \mathrm{~kb}$ ) 
Additional file 8: Figure S5. Histogram presentation of clusters of orthologous groups (COGs) classification of unigenes (A) and differentially expressed genes in WW-transformants compared with wild-type hop (B). (JPG 469 kb)

Additional file 9: Table S4. Transcription factors identified in the transcriptome of hop. (XLS $106 \mathrm{~kb}$ )

Additional file 10: Figure S6. Distribution of top 11 identified transcription factors from hop unigenes into transcription factor families. (JPG $165 \mathrm{~kb}$ )

Additional file 11: Table S5. The unigenes differentially expressed between the control and WW-transgenic plants of hop. (XLS 296 kb)

Additional file 12: Figure S7. HPLC analysis of menthanolic extracts of leaves of WT and WW-transgenic hop. Quantification (\% DM) of (A) Gallic acid (phenolic acids), (B) a-bitter acids, and (C) xanthohumol was performed using their respective working standards. The graph shows values \pm SD of three leaves from B11, B22, and B24 transgenic lines of the hop. (JPG $111 \mathrm{~kb})$

\section{Abbreviations}

4CL: 4-coumaroyl CoA-Ligase; BA: bitter acids; C4H: cinnamate 4-hydroxylase; CHS_H1: "true" chalcone synthase; OMT1: O-methyltransferase 1; PAL: phenylalanine ammonia-lyase; PF: prenylflavonoid; PRT: prenyltransferase; qRT-PCR: quantitative real-time PCR, WT, wild-type; WW-lines: HIWRKY1 and HIWDR1 overexpressing lines

\section{Acknowledgments}

We apologize to the many colleagues whose work could not be cited in this manuscript due to space limitation. We would like to thank Helena Matoušková, Olga Horáková, Kristýna Siglová and Anna Lomnická from Biology Centre of the CAS, Institute of Plant Molecular Biology (IPMB) for excellent technical assistance and Petr Svoboda (HRI) for maintenance of the transformed hop lines. We are highly grateful to Prof. Csaba Koncz from the Max Planck Institute for Plant Breeding, Cologne (Germany) for providing the pPCV91 vector plasmid for Agrobacterium transformation.

\section{Funding}

We acknowledge the financial support received from the Czech Science Foundation GACR 13-03037S and institutional support RVO:60077344. The funding bodies did not have any role in the design of the study and collection, analysis, and interpretation of data and in writing the manuscript.

\section{Availability of data and materials}

The dataset(s) supporting the conclusions of this article are included within the article and its additional file(s). The complete raw RNA-seq datasets have been deposited in the NCBI-SRA repository under accession number SRR6308266, SRR6308267, SRR6308195, and SRR6308265.

\section{Authors' contributions}

AKM: performed bioinformatics works, analyzed the data, participated in experiments and wrote the manuscript. GSD and Jj: participated in bioinformatics analysis and proof reading of the article. GSD: performed blotting experiment. MK performed total RNA isolation and GRT-PCR analysis. TK, AKM, and JB: performed transformation of hop. JP: responsible for maintenance and surveillance of transformant lines. TS: generated the biological replicate of RNA sequencing data. JM: constructed the plant expression vector, designed and coordinated the experiments, coordinated the GACR project and proofreading of the article. All authors have read and approved the final manuscript.

\section{Ethics approval and consent to participate}

No permission was required from the ethics committee as the project did not involve testing of human, animal or endangered plant species subjects All experiments with transgenic plants were performed in accordance with the national law and institutional guidelines of Biology Centre of the Czech Academy of Sciences.

\section{Consent for publication}

Not applicable.

\section{Competing interests}

The authors declare that they have no competing interests.

\section{Publisher's Note}

Springer Nature remains neutral with regard to jurisdictional claims in published maps and institutional affiliations.

\section{Author details}

${ }^{1}$ Biology Centre of the Czech Academy of Sciences, Institute of Plant Molecular Biology, Department of Molecular Genetics, Branišovská 31, 37005 České Budějovice, Czech Republic. ²Department of Agronomy, Biotechnical Faculty, University of Ljubljana, Jamnikarjeva 101, SI-1000 Ljubljana, Slovenia. ${ }^{3}$ Hop Research Institute, Co. Ltd., Kadaňská 2525, 43846 Žatec, Czech Republic. ${ }^{4}$ Faculty of Agriculture and Life Science, Department of Applied Biosciences, Hirosaki University, Hirosaki, Aomori 036-8561, Japan.

Received: 21 November 2017 Accepted: 27 September 2018 Published online: 11 October 2018

\section{References}

1. Lamy V, Roussi S, Chaabi M, Gossé F, Lobstein A, Raul F. Lupulone, a hop bitter acid, activates different death pathways involving apoptotic TRAILreceptors, in human colon tumor cells and in their derived metastatic cells. Apoptosis. 2008:13(10):1232-42.

2. Hougee S, Faber J, Sanders A, Berg WB, Garssen J, Smit HF, Hoijer MA. Selective inhibition of $\mathrm{COX}-2$ by a standardized $\mathrm{CO} 2$ extract of Humulus lupulus in-vitro and its activity in a mouse model of zymosan-induced arthritis. Planta Med. 2006;72(3):228-33.

3. Overk CR, Yao P, Chadwick LR, Nikolic D, Sun Y, Cuendet MA, Deng Y, Hedayat AS, Pauli GF, Farnsworth NR, van Breemen RB, Bolton JL. Comparison of the in vitro estrogenic activities of compounds from hops (Humulus lupulus) and red clover (Trifolium pratense). J Agric Food Chem. 2005;53(16):6246-53.

4. Franco L, Sánchez CL, Bravo R, Rodríguez AB, Barriga C, Cubero J. The sedative effects of hops (Humulus lupulus), a component of beer, on the activity/rest rhythm. Acta Physiol Hung. 2012:99(2):133-9.

5. Rozalski M, Micota B, Sadowska B, Stochmal A, Jedrejek D, WieckowskaSzakiel M, Rozalska B. Antiadherent and Antibiofilm activity of Humulus lupulus L. derived products: new pharmacological properties. Biomed Res Int. 2013;2013:101089.

6. Liu Y, Gu X, Tang J, Liu K. Antioxidant activities of hops (Humulus lupulus) and their products. J Am Soc Brew Chem. 2007;65(2):116-21.

7. Roberts TR, Wilson RJH. Handbook of brewing. In: Priest FJ, Stewart GG, editors. 2nd edn. Boca Raton: Hops. Taylor \& Francis; 2006. p. 177-280

8. Patzak J, Krofta K, Henychová A, Nesvadba V. Number and size of lupulin glands, glandular trichomes of hop (Humulus lupulus L.) play key-role in contents of bitter acids and polyphenols in hop cone. Int J Food Sci Technol. 2015;50(9):1864-72.

9. De Keukeleire J, Ooms G, Heyerick A, Roldan-Ruiz I, Van Bockstaele E, De Keukeleire D. Formation and accumulation of alpha-acids, beta-acids, desmethylxanthohumol, and xanthohumol during flowering of hops (Humulus lupulus L.). J Agric Food Chem. 2003;51(15):4436-41.

10. Wang G, Tian L, Aziz N, Broun P, Dai X, He J, King A, Zhao PX, Dixon RA. Terpene biosynthesis in glandular trichomes of hop. Plant Physiol. 2008;148(3):1254-66.

11. Nagel J, Culley LK, Lu Y, Liu E, Matthews PD, Stevens JF, Page JE. EST analysis of hop glandular trichomes identifies an O-methyltransferase that catalyzes the biosynthesis of xanthohumol. Plant Cell. 2008:20(1):186-200.

12. Sägesser M, Deinzer M. HPLC-ion spray tandem mass spectrometry of flavonol glycosides in hops. J Am Soc Brew Chem. 1996:54(3):129-34

13. McAdam EL, Vaillancourt RE, Koutoulis A, Whittock SP. Quantitative genetic parameters for yield, plant growth and cone chemical traits in hop (Humulus lupulus L.). BMC Genet. 2014;15(2):1-18.

14. Vogt T. Phenylpropanoid biosynthesis. Mol Plant. 2010;3(1):2-20.

15. Matoušek J, Vrba L, Škopek J, Orctová L, Pešina K, Heyerick A, Baulcombe D, De Keukeleire, D. Sequence analysis of a "true chalcone synthase (chs_ $\mathrm{H1}$ ) oligofamily from hop (Humulus lupulus L) and PAP1 activation of chs H1 in heterologous systems. J Agric Food Chem 2006a;54(20):7606-7615.

16. Tsurumaru Y, Sasaki K, Miyawaki T, Momma T, Umemoto N, Yazaki K. An aromatic prenyltransferase-like gene HIPT-1 preferentially expressed in lupulin glands of hop. Plant Biotech. 2010;27(2):199-204.

17. Matoušek J, Kocábek T, Patzak J, Stehlík J, Füssy Z, Krofta K, Heyerick A, Roldán-Ruiz I, Maloukh L, De-Keukeleire D. Cloning and molecular analysis of HIbZip1 and HlbZip2 transcription factors putatively involved in the 
regulation of the lupulin metabolome in hops (Humulus lupulus L.). J Agric Food Chem. 2010;58(2):902-12.

18. Matoušek J, Kocábek T, Patzak J, Füssy Z, Procházková J, Heyerick A. Combinatorial analysis of lupulin gland transcription factors from R2R3Myb, bHLH and WDR families indicates a complex regulation of chs_H1 genes essential for prenylflavonoid biosynthesis in hop (Humulus lupulus L.). BMC Plant Biol. 2012;20(12):27

19. Matoušek J, Kocábek T, Patzak J, Bríza J, Siglová K, Mishra AK, Duraisamy GS, Týcová A, Ono E, Krofta K. The "putative" role of transcription factors from HIWRKY family in the regulation of the final steps of prenylflavonoid and bitter acids biosynthesis in hop (Humulus lupulus L.). Plant Mol Biol. 2016; 92(3):263-77.

20. Kocábek T, Mishra AK, Matoušek J, Patzak J, Lomnická A, Khare M, Krofta K. The R2R3 transcription factor HIMYB8 and its role in flavonoid biosynthesis in hop (Humulus lupulus L.). Plant Sci. 2018;269:32-46.

21. Devaiah BN, Karthikeyan AS, Raghothama KG. WRKY75 transcription factor is a modulator of phosphate acquisition and root development in Arabidopsis. Plant Physiol. 2007;143(4):1789-801.

22. Zhang B, Schrader A. TRANSPARENT TESTA GLABRA 1-dependent regulation of flavonoid biosynthesis. Plants (Basel). 2017;6(4):E65.

23. Jakše J, Kindlhofer K, Javornik B. Assessment of genetic variation and differentiation of hop genotypes by microsatellite and AFLP markers. Genome. 2001;44(5):773-82.

24. Murakami A, Darby P, Javornik B, Pais MS, Seigner E, Lutz A, Svoboda P. Molecular phylogeny of wild hops, (Humulus lupulus L.). Heredity. 2006;97(1):66-74.

25. Gatica-Arias A, Stanke M, Hantzschel KR, Matousek J, Weber G. Overexpression of the transcription factor HIMYB3 in transgenic hop (Humulus lupulus L. Cv. Tettnanger) modulates the expression of genes involved in the biosynthesis of flavonoids and phloroglucinols. Plant Cell Tiss Org. 2013;113: 279-89.

26. Zhong GY. Genetic issues and pitfalls in transgenic plant breeding. Euphytica. 2001;118(2):137-44.

27. Koetle MJ, Finnie JF, Balázs E, Van Staden J. A review on factors affecting the Agrobacterium-mediated genetic transformation in ornamental monocotyledonous geophytes. S Afr J Bot. 2015;98:37-44.

28. Luo J, Butelli E, Hill L, Par A, Niggeweg R, Bailey P, Weisshaar B, et al. AtMYB12 regulates caffeoyl quinic acid and flavonol synthesis in tomato: expression in fruit results in very high levels of both types of polyphenols. Plant J. 2008:56(2):316-26.

29. Pandey A, Misra P, Bhambhani S, Bhatia C, Trivedi PK. Expression of Arabidopsis MYB transcription factor, AtMYB111, in tobacco requires light to modulate flavonol content. Sci Rep. 2014;21(4):5018.

30. Pandey A, Misra P, Choudhary D, Yadav R, Goel R, Bhambhani S, Sanyal I, Trivedi I, Trivedi PK. AtMYB12 expression in tomato leads to large-scale differential modulation in transcriptome and flavonoid content in leaf and fruit tissues. Sci Rep. 2015;24(5):12412.

31. Lambirth KC, Whaley AM, Blakley IC, Schlueter JA, Bost KL, Loraine AE, Pille KJ. A comparison of transgenic and wild-type soybean seeds: analysis of transcriptome profiles using RNA-Seq. BMC Biotechnol. 2015;1(15):89.

32. Ricroch $A E$, Berge JB, Kuntz M. Evaluation of genetically engineered crops using transcriptomic, proteomic, and metabolomic profiling techniques. Plant Physiol. 2011;155(4):1752-61.

33. Metzker ML. Sequencing technologies - the next generation. Nat Rev Genet. 2010;11(1):31-46.

34. Alagna F, D'Agostino N, Torchia L, Servili M, Rao R, Pietrella M, Giuliano G, et al. Comparative 454 pyrosequencing of transcripts from two olive genotypes during fruit development. BMC Genomics. 2009;26(10):399-414.

35. Guo S, Zheng Y, Joung JG, Liu S, Zhang Z, Crasta OR, Sobral BW, Xu Y, Huang S, Fei Z. Transcriptome sequencing and comparative analysis of cucumber flowers with different sex types. BMC Genomics. 2010;17(11):384.

36. Garg R, Patel RK, Jhanwar S, Priya P, Bhattacharjee A, Yadav G, Bhatia S, Chattopadhyay D, Tyagi AK, Jain M. Gene discovery and tissue-specific transcriptome analysis in chickpea with passively parallel pyrosequencing and web resource development. Plant Physiol. 2011;156(4):1661-78.

37. Alba R, Payton P, Fei Z, Mc Quinn R, Debbie P, Martin GB, Tanksley SD, Giovannoni JJ. Transcriptome and selected metabolite analyses reveal multiple points of ethylene control during tomato fruit development. Plant Cell. 2005;17(11):2954-65.

38. Massa AN, Childs KL, Lin H, Bryan GJ, Giuliano G, Buell CR. The transcriptome of the reference potato genome Solanum tuberosum group Phureja clone DM1-3 516R44. PLoS One. 2011;6(10):e26801.
39. Li R, Zhai H, Kang C, Liu D, He S, Liu Q. De Novo transcriptome sequencing of the orange-fleshed sweet potato and analysis of differentially expressed genes related to carotenoid biosynthesis. Int J Genomics. 2015;2015:843802.

40. Dunwell JM, Moya-León MA, Herrera R. Transcriptome analysis and crop improvement (a review). Biol Res. 2001;34(3-4):153-64.

41. Pérez-de-Castro AM, Vilanova S, Canizares J, Pascual L, Blanca JM, Diez MJ, Prohens J, Picó B. Application of genomic tools in plant breeding. Curr Genomics. 2012;13(3):179-95.

42. Ward JA, Ponnala L, Weber CA. Strategies for transcriptome analysis in nonmodel plants. Am J Bot. 2012;99(2):267-76.

43. Coll A, Nadal A, Collado R, Capellades G, Kubista M, Messeguer J, Pla M. Natural variation explains most transcriptomic changes among maize plants of MON810 and comparable non-GM varieties subjected to two Nfertilization farming practices. Plant Mol Biol. 2010;73(3):349-62.

44. Abdeen A, Schnell J, Miki B. Transcriptome analysis reveals absence of unintended effects in drought-tolerant transgenic plants overexpressing the transcription factor ABF3. BMC Genomics. 2010;11:69.

45. Vojta P, Kokáš F, Husičková A, Grúz J, Bergougnoux V, Marchetti CF, Jiskrová E, Ježilová E, Mik V, Ikeda Y, Galuszka P. Whole transcriptome analysis of transgenic barley with altered cytokinin homeostasis and increased tolerance to drought stress. New Biotechnol. 2016;33(5):676-91.

46. Jiang Q, Niu F, Sun X, Hu Z, Li X, Ma Y, Zhang H. RNA-seq analysis of unintended effects in transgenic wheat overexpressing the transcription factor GmDREB1. Crop J. 2017:5(3):207-18.

47. Shen WJ, Forde BG. Efficient transformation of Agrobacterium sp. by high voltage electroporation. Nucleic Acids Res. 1989;17(20):8385.

48. Roy AT, Leggett $G$, Koutoulis A. Development of a shoot multiplication system for hop (Humulus lupulus L.). In vitro Cell Dev Biol. 2001;37(1):79-83.

49. Tai TH, Tanksley SD. A rapid and inexpensive method for isolation of total DNA from dehydrated plant tissue. Plant Mol Biol Rep. 1990;8(4):297-303.

50. Church GM, Gilbert W. Genomic sequencing. Proc Natl Acad Sci U S A. 1984:81(2):1991-5.

51. Lichtenthaler HK, Wellburn AR. Determinations of total carotenoids and chlorophylls $\mathrm{a}$ and $\mathrm{b}$ of leaf extracts in different solvents. Biochem Soc Trans. 1983;11:591-2.

52. Bolger AM, Lohse M, Usadel B. Trimmomatic: a flexible trimmer for Illumina sequence data. Bioinformatics. 2014;30(15):2114-20.

53. Anders $\mathrm{S}$, Huber W. Differential expression analysis for sequence count data. Genome Biol. 2010;11(10):R106

54. Natsume S, Takagi H, Shiraishi A, Murata J, Toyonaga H, Patzak J, Takagi M, Yaegashi H, Uemura A, Mitsuoka C, Yoshida K, Krofta K, Satake H, Terauchi R, Ono E. The draft genome of hop (Humulus lupulus), an essence for brewing. Plant Cell Physiol. 2014;56(3):428-41.

55. Iwata $\mathrm{H}$, Gotoh $\mathrm{O}$. Benchmarking spliced alignment programs including Spaln2, an extended version of Spaln that incorporates additional speciesspecific features. Nucleic Acids Res. 2012;40(20):e161.

56. Simao FA, Waterhouse RM, loannidis P, Kriventseva EV, Zdobnov EM. BUSCO: assessing genome assembly and annotation completeness with single-copy orthologs. Bioinformatics. 2015;31:3210-2.

57. Conesa A, Gotz S. Blast2GO: a comprehensive suite for functional analysis in plant genomics. Int J Plant Genomics. 2008;2008:619832.

58. Tatusov RL, Galperin MY, Natale DA, Koonin EV. The COG database: a tool for genome-scale analysis of protein functions and evolution. Nucleic Acids Res. 2000;28(1):33-6.

59. Moriya Y, Itoh M, Okuda S, Yoshizawa AC, Kanehisa M. KAAS: an automatic genome annotation and pathway reconstruction server. Nucleic Acids Res. 2006;35(Web Server issue):182-5.

60. Dai X, Sinharoy S, Udvardi M, Zhao PX. PlantTFcat: an online plant transcription factor and transcriptional regulator categorization and analysis tool. BMC Bioinformatics. 2013;12(14):321.

61. Grabherr MG, Haas BJ, Yassour M, Levin JZ, Thompson DA, Amit I, Adiconis X, Fan L, Raychowdhury R, Zeng Q, Chen Z, Mauceli E, Hacohen N, Gnirke A, Rhind N, di Palma F, Birren BW, Nusbaum C, Lindblad-Toh K, Friedman N, Regev A. Full-length transcriptome assembly from RNA-Seq data without a reference genome. Nat Biotechnol. 2011;29:644-52.

62. Love Ml, Huber W, Anders S. Moderated estimation of fold change and dispersion for RNA-seq data with DESeq2. Genome Biol. 2014;15(12):550

63. Zhao S, Guo Y, Sheng Q, Shyr Y. Advanced heat map and clustering analysis using heatmap3. Biomed Res Int. 2014;2014:986048.

64. Du Z, Zhou X, Ling Y, Zhang ZH, Su Z. AgriGO: a GO analysis toolkit for the agricultural community. Nucleic Acids Res. 2010;38:W64-70. 
65. Thimm O, Bläsing O, Gibon Y, Nagel A, Meyer S, Krüger P, Selbig J, Müller LA, Rhee SY, Stitt M. MAPMAN: a user-driven tool to display genomics data sets onto diagrams of metabolic pathways and other biological processes. Plant J. 2004;37(6):914-39.

66. Finn RD, Bateman A, Clements J, Coggill P, Eberhardt RY, Eddy SR, Heger A, Hetherington K, Holm L, Mistry J, Sonnhammer EL, Tate J, Punta M. Pfam: the protein families database. Nucleic Acids Res. 2014;42:D222-30.

67. Maloukh L, Matousek J, Van-Bockstaele E, Roldán-Ruiz I. Housekeeping gene selection for real time-PCR normalization in female hop (Humulus lupulus L) tissues. J Plant Biochem Biotechnol. 2009;18(1):53-8.

68. Livak KJ, Schmittgen TD. Analysis of relative gene expression data using real-time quantitative $P C R$ and the 2(-Delta Delta $C(T))$ method. Methods. 2001;25(4):402-8.

69. Matoušek J, Vrba L, Škopek J, Orctová L, Pešina K, Heyerick A, Baulcombe D, De Keukeleire D. Sequence analysis of a 'true' chalcone synthase (chs_H1) oligofamily from hop (Humulus lupulus L.) and PAP1 activation of chs_H1 in heterologous systems. J Agric Food Chem. 2006b;54:7606-15.

70. Gupta P, Goel R, Pathak S, Srivastava A, Singh SP, Sangwan RS, Asif MH, Trivedi PK. De novo assembly, functional annotation and comparative analysis of Withania somnifera leaf and root transcriptomes to identify putative genes involved in the withanolides biosynthesis. PLoS One. 2013; 8(5):62714.

71. Wang Z, Fang B, Chen J, Zhang X, Luo Z, Huang L, Chen X, Li Y. De novo assembly and characterization of root transcriptome using Illumina paired end sequencing and development of cSSR markers in sweet potato (Ipomoea batatas). BMC Genomics. 2010;24(11):726.

72. Novaes E, Drost DR, Farmerie WG, Pappas GJ, Grattapaglia D, Sederoff RR, Kirst M. High-throughput gene and SNP discovery in Eucalyptus grandis, an uncharacterized genome. BMC Genomics. 2008;30(9):312-0.

73. Garzón-Martínez GA, Zhu Zl, Landsman D, Barrero LS, Mariño-Ramírez L. The Physalis peruviana leaf transcriptome: assembly, annotation and gene model prediction. BMC Genomics. 2012;25(13):151-10.

74. Han R, Takahashi H, Nakamura M, Bunsupa S, Yoshimoto N, Yamamoto H, Suzuki H, Shibata D, Yamazaki M, Saito K. Transcriptome analysis of nine tissues to discover genes involved in the biosynthesis of active ingredients in Sophora flavescens. Biol Pharm Bull. 2015;38(6):876-83.

75. Jain M, Misra G, Patel RK, Priya P, Jhanwar S, Khan AW, Shah N, Singh VK, Garg R, Jeena G, Yadav M, Kant C, Sharma P, Yadav G, Bhatia S, Tyagi AK, Chattopadhyay D. A draft genome sequence of the pulse crop chickpea (Cicer arietinum L.). Plant J. 2013;74(5):715-29.

76. Xu C, Jiao C, Zheng Y, Sun H, Liu W, Cai X, Wang X, Liu S, Xu Y, Mou B, Dai S, Fei Z, Wang $Q$. De novo and comparative transcriptome analysis of cultivated and wild spinach. Sci Rep. 2015;5:17706

77. Severin AJ, Woody JL, Bolon YT, Joseph B, Diers BW, Farmer AD, Muehlbauer GJ. RNA-Seq atlas of Glycine max: a guide to the soybean transcriptome. BMC Plant Biol. 2010;10:160.

78. Pokorn T, Radišek S, Javornik B, Štajner N, Jakše J. Development of hop transcriptome to support research into host-viroid interactions. PLoS One. 2017;12(9):e0184528

79. Wei W, Qi X, Wang L, Zhang Y, Hua W, Li D, Lv H, Zhang X. Characterization of the sesame (Sesamum indicum L.) global transcriptome using Illumina paired-end sequencing and development of EST-SSR markers. BMC Genomics. 2011;12:451.

80. Goyal E, Amit SK, Singh RS, Mahato AK, Chand S, Kanika K. Transcriptome profiling of the salt-stress response in Triticum aestivum cV. Kharchia Local Sci Rep. 2016;13(6):27752.

81. Čeh B, Kač M, Košir IJ, Abram V. Relationships between Xanthohumol and polyphenol content in hop leaves and hop cones with regard to water supply and cultivar. Int J Mol Sci. 2007;8:989-1000.

82. Prencipe FP, Brighenti V, Rodolfi M, Mongelli A, dall'Asta C, Ganino T, Bruni $R$, Pellati $F$. Development of a new high-performance liquid chromatography method with diode array and electrospray ionization-mass spectrometry detection for the metabolite fingerprinting of bioactive compounds in Humulus lupulus L. J Chromatogr A. 2014;1349:50-9.

83. Krause E, Yuan Y, Hajirahimkhan A, Dong H, Dietz BM, Nikolic D, Pauli GF, Bolton $J L$, van Breemen RB. Biological and chemical standardization of a hop (Humulus lupulus) botanical dietary supplement. Biomed Chromatogr. 2014;28(6):729-34

84. Jackowski J, Hurej M, Roj E, Poplonski J, Kosny L, Huszcza E. Antifeedant activity of xanthohumol and supercritical carbon dioxide extract of spent hops against stored product pests. Bull Entomol Res. 2015;105(4):456-61.
85. Gatica-Arias A, Farag MA, Stanke M, Matoušek J, Wessjohann L, Weber G. Flavonoid production in transgenic hop (Humulus lupulus L.) altered by PAP1/MYB75 from Arabidopsis thaliana L. Plant Cell Rep. 2012;31(1):111-9.

86. Kavalier AR, Ma CH, Figueroa M, Kincaid D, Matthews PD, Kennelly EJ. Targeted analysis of polyphenol metabolism during development of hop (Humulus lupulus L) cones following treatment with prohexadione-calcium. Food Chem. 2014;15(145):254-63.

87. Butelli E, Titta L, Giorgio M, Mock HP, Matros A, Peterek S, Schijlen EG, Hall RD, Bovy AG, Luo J, Martin C. Enrichment of tomato fruit with healthpromoting anthocyanins by expression of select transcription factors. Nat Biotechnol. 2008;26:1301-8.

88. Leonard E, Lim KH, Saw PN, Koffas MA. Engineering central metabolic pathways for high-level flavonoid production in Escherichia coli. Appl Environ Microbiol. 2007;73(12):3877-86.

89. Zhang YHP. Substrate channeling and enzyme complexes for biotechnological applications. Biotechnol Adv. 2011;29(6):715-25.

90. Reddy PM, Rendón-Anaya M, de los Dolores Soto del Rio M, Khandual S. Flavonoids as signaling molecules and regulators of root nodule development. Dynamic Soil Dynamic Plant 2007;1:83-94.

91. Nakabayashi R, Yonekura-Sakakibara K, Urano K, Suzuki M, Yamada Y, Nishizawa T, Matsuda F, Kojima M, Sakakibara H, Shinozaki K, Michael AJ, Tohge T, Yamazaki M, Saito K. Enhancement of oxidative and drought tolerance in Arabidopsis by over-accumulation of antioxidant flavonoids. Plant J. 2014;77:367-79.

92. Bourque S, Dutartre A, Hammoudi V, Blanc S, Dahan J, Jeandroz S, Pichereaux C, Rossignol M, Wendehenne D. Type-2 histone deacetylases as new regulators of elicitor-induced cell death in plants. New Phytol. 2011; 192(1):127-39.

93. Latrasse D, Jégu T, Li H, de Zelicourt A, Raynaud C, Legras S, Gust A, Samajova O, Veluchamy A, Rayapuram N, Ramirez-Prado JS, Kulikova O, Colcombet J, Bigeard J, Genot B, Bisseling T, Benhamed M, Hirt H. MAPKtriggered chromatin reprogramming by histone deacetylase in plant innate immunity. Genome Biol. 2017;18(1):131.

94. Zhao Y. Auxin biosynthesis and its role in plant development. Annu Rev Plant Biol. 2010;61:49-64.

95. Lackman P, González-Guzmán M, Tilleman S, Carqueijeiro I, Pérez AC, Moses T, Seo M, Kanno Y, Häkkinen ST, Van Montagu MC, Thevelein JM, Maaheimo H, Oksman-Caldentey KM, Rodriguez PL, Rischer H, Goossens A. Jasmonate signaling involves the abscisic acid receptor PYL4 to regulate metabolic reprogramming in Arabidopsis and tobacco. Proc Natl Acad Sci U S A. 2011; 108(14):5891-6.

96. Hemm MR, Rider SD, Ogas J, Murry DJ, Chapple C. Light induces phenylpropanoid metabolism in Arabidopsis roots. Plant J. 2004;38(5):765-78.

97. Zoratti L, Karppinen K, Luengo Escobar A, Häggman H, Jaakola L. Lightcontrolled flavonoid biosynthesis in fruits. Front Plant Sci. 2014;9(5):534.

98. Wade HK, Bibikova TN, Valentine WJ, Jenkins Gl. Interactions within a network of phytochrome, cryptochrome and UV-B phototransduction pathways regulate chalcone synthase gene expression in Arabidopsis leaf tissue. Plant J. 2001;25(6):675-85.

99. Giliberto L, Perrotta G, Pallara P, Weller JL, Fraser PD, Bramley PM, Fiore A, Tavazza M, Giuliano G. Manipulation of the blue light photoreceptor cryptochrome 2 in tomato affects vegetative development, flowering time, and fruit antioxidant content. Plant Physiol. 2005;137(1):199-208.

100. Rushton PJ, Somssich IE, Ringler P, Shen QJ. WRKY transcription factors. Trends Plant Sci. 2010;15(5):247-58.

101. Duraisamy GS, Mishra AK, Kocabek T, Matoušek J. Identification and characterization of promoters and cis-regulatory elements of genes involved in secondary metabolites production in hop (Humulus lupulus. L). Comput Biol Chem. 2016:64:346-52.

102. Wegner A, Meiser J, Weindl D, Hiller K. How metabolites modulate metabolic flux. Curr Opin Biotechnol. 2015;34:16-22.

103. Phukan UJ, Jeena GS, Shukla RK. WRKY transcription factors: molecular regulation and stress responses in plants. Front Plant Sci. 2016;7:760.

104. Feller A, Machemer K, Braun EL, Grotewold E. Evolutionary and comparative analysis of MYB and bHLH plant transcription factors. Plant J. 2011;66(1):94-116. 